\title{
The barrier to genetic exchange between hybridising populations
}

\author{
Nick Barton* and \\ Bengt Olle Bengtsson $\dagger$
}

* Department of Genetics and Biometry, University
College London, 4 Stephenson Way, London
NW1 2HE, U.K.
† Department of Genetics, University of Lund,
Sölvegatan 29, S-223 62 Lund, Sweden.

Suppose that selection acts at one or more loci to maintain genetic differences between hybridising populations. Then, the flow of alleles at a neutral marker locus which is linked to these selected loci will be impeded. We define and calculate measures of the barrier to gene flow between two distinct demes, and across a continuous habitat. In both cases, we find that in order for gene flow to be significantly reduced over much of the genome, hybrids must be substantially less fit, and the number of genes involved in building the barrier must be so large that the majority of other genes become closely linked to some locus which is under selection. This conclusion is not greatly affected by the pattern of epistasis, or the position of the marker locus along the chromosome.

\section{INTRODUCTION}

The separation of biological species requires the evolution of genetic barriers to gene exchange. Thus, to understand the process of speciation, we need to know not only how genetic differences evolve, but also the effect these differences have on gene flow. Genetic differences can only lead to significant reproductive isolation if they either directly cause a substantial reduction in gene flow at the majority of loci, or if they facilitate the evolution of further genetic differences which eventually produce a strong barrier to gene flow. Here, we only consider the first, direct question: what effect do genetic differences have on gene flow at other loci?

The simplest type of genetic barrier is formed when selection acts at a single locus. If two populations carry different alleles at this locus, and the alleles of each population are selected against in the environment or genetic background of the other population, then this genetic difference will act as a barrier to gene flow at other loci. A similar situation arises if, for example, the two populations differ by a chromosomal translocation that causes some sterility in heterozygotes. When genes at other loci migrate into the new population, they will be associated with disadvantageous alleles at the selected locus, and so will tend to be eliminated, even if they are themselves selectively neutral. This selection pressure will only cease after the incoming alleles have recombined away from their old genetic background. The rate of flow of a neutral marker into the new population will therefore depend on the ratio between selection at the "barrier locus", and recombination between that locus and the marker locus.

This simple case has been analysed mathematically by Bengtsson $(1974,1985)$ and Spirito et al. (1983), who considered exchange between two discrete demes, and by Barton (1979) and Petry (1983), who considered flow through a continuous habitat. All these authors conclude that a single genetic difference can only present a substantial barrier to the flow of those genes which are so tightly linked to the selected locus that selection is much stronger than the rate of recombination. Since most genes will not be linked to the single selected locus, this implies that the fitness of first or second generation hybrids must be very low if the flow of most genes in the organism is to be impeded. Barton (1979) has, moreover, shown that even if the barrier is strong enough that linked neutral alleles remain differentiated for many generations, alleles with even a slight selective advantage will quickly penetrate the barrier. These properties of single locus barriers make it unlikely that the fixation of a chromosomal rearrangement could trigger the divergence of a new species through its effect on gene flow, as suggested 
by White $(1968,1978)$ in his "stasipatric" model.

These results do not, however, allow us to answer more general questions about speciation. Reproductive isolation between even closely related species is usually based on many genes (Dobzhansky, 1937; Charlesworth et al., 1982; Maynard Smith, 1983; Barton and Charlesworth, 1984), and hybrid zones are usually maintained by selection on many loci (Barton and Hewitt, 1985). We must therefore know the strength of the barrier to gene flow which is set up by selection on many genes before we can interpret these observations. Bengtsson (1985) has shown that for multiplicative fitness interactions between the genetic loci which maintain the barrier, the number of loci makes little difference to the flow of a neutral marker allele, provided that the fitness of the first generation hybrids is kept constant, and the marker is unlinked to any of the loci maintaining the barrier. As the number of loci increases, this last assumption becomes unrealistic, since the marker locus will then most likely be linked to some of the loci maintaining the barrier. Barton (1983) and Barton and Hewitt (1983) have studied this situation in a model of a continuous habitat, also using multiplicative fitnesses, and found that with this model, selection on a large number of linked loci gives a stronger barrier than when the same selection pressure is concentrated on a single locus.

Here, we will extend these studies on the flow of a neutral marker allele through a genetic barrier involving many loci. Before starting the analysis, however, we will define some of the terms that are of importance in the paper. In particular, we will attempt to define the "effective migration rate" between genetically distinct populations, and the "strength" of genetic barriers in such a way that these terms can be used consistently across a variety of models. The technical core of our argument, which contains the methods needed to derive numerical values for barrier strength, is set out in the appendix. These methods are applied to investigate the dependence of gene flow on various parameters, and are explained by heuristic analysis of some limiting cases.

\section{DEFINITIONS}

The concept of "gene flow" has a clear intuitive meaning when two geographically separate populations are regarded and where a direct exchange of migrants occurs between the populations. If there are no selective interactions between the genes in the two populations, the best way of measuring the gene flow from population 2 into population 1 is by the migration rate between the two populations. This migration rate we define as the number of individuals born in population 2 that exists in population 1 after migration, divided by the number in population 1 after migration. The migration and thus the gene flow from one population to another may well be different from the migration and gene flow going in the other direction. The magnitude of the gene flow between the populations can be studied by following its effect on a genetic difference between the two populations. Consider a marker allele which is selectively neutral in both populations, and which has frequencies $u_{1}$ and $u_{2}$ in the populations before migration (fig. 1(a)). The change in frequency, $\delta u$, of the allele in population 1 due to migration from population 2 can then be written:

$$
\delta u=m \Delta u .
$$

Here, $\Delta u\left(=u_{2}-u_{1}\right)$ is the difference in allele frequencies between the two populations, and $m$ is the migration rate from 2 into 1 , as defined above. The change in frequency per generation due to migration, $\delta u$, will be called the influx from 2 into 1. Eq. 1 alone predicts a persistent change in $u$. However, we now suppose that this influx is balanced by an equal and opposite efflux, $-\delta u$, so that the system remains at equilibrium. This efflux might, for example, be to other populations at still lower allele frequencies (fig. 1(a)).

This simple idea of flux can help us give a meaning to the concept of gene flow between two populations when their relationship is less clear than in the situation just described. However, it must first be extended to cover the case where the populations differ in size. Then, we define $u_{i}$ as the number of genes in population $i$, and $\delta u_{i}$ as the rate of change of numbers of genes in $i$ due to migration. The $u_{i}$ and $\delta u_{i}$ may, of course, be multiplied throughout by any convenient normalising constant; in the cases we consider, the initial and final populations $(1,2$ below) will be the same size, and so $u_{1}$ and $u_{2}$ (but not $u_{i}, i \neq 1,2$ ) are taken to be the frequencies in 1 and 2 .

Consider two populations embedded in a complex network of interconnected populations. One can still think of an indirect flow of genes between them, even though they may not exchange any direct migrants at all (fig. 1(b)). To define what will be called an effective migration rate between them, assume that they differ at a selectively neutral locus just as before, such that $\Delta u=u_{2}-u_{1}$. Assume also that there is a constant outflow of this allele 
(a)

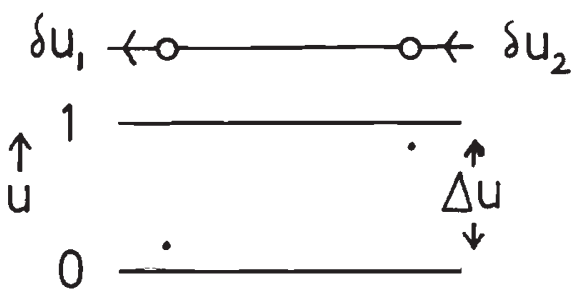

(b)
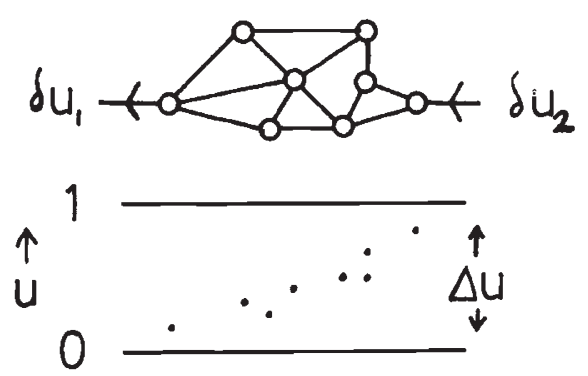

(c)
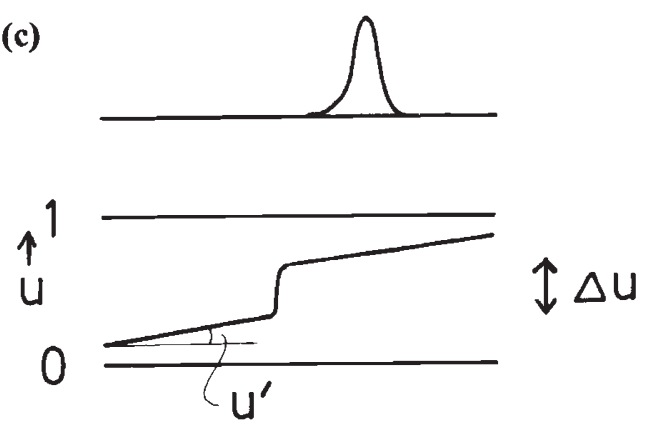

(d)

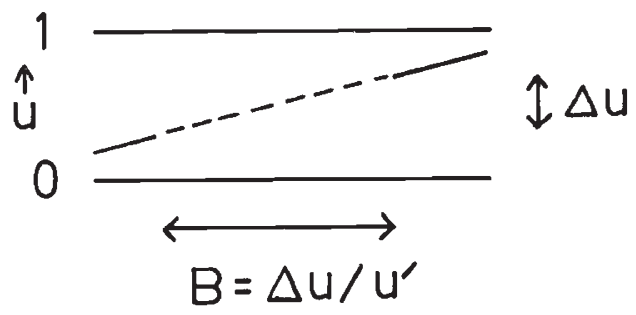

Figure 1 (a) Two demes (represented by open circles) exchange migrants directly. A flux of neutral alleles, $\delta u_{2}$, enters deme 2 , on the right, whilst a flux $\delta u_{1}$ leaves deme 1 , on the left. These fluxes maintain a difference in allele frequency $\Delta u$ between the populations (lower diagram). (b) Two demes exchange migrants indirectly, via a network of interconnected demes. As in (a), fluxes $\delta u_{1}$ and $\delta u_{2}$ maintain a stable difference in allele frequency, $\Delta u$ (lower diagram). The effective migration rate into deme 1 is defined as $m_{e}=\delta u_{1} / \Delta u$. (c) In a continuous, one-dimensional population, the matrix of migration rates between discrete demes is replaced by the distribution of distances between parents and offspring (upper diagram). The movement of genes along the transect can now be approximated by diffusion (Eq. 3). A localised barrier to gene flow will produce a step in the frequency of a neutral allele, $\Delta u$, proportional to the gradient $\partial u / \partial x \equiv u^{\prime}$ (lower diagram) away from the barrier. The strength of the barrier is defined as $B \equiv \Delta u / u^{\prime}$. (d) This barrier strength has the dimensions of a distance; it can be thought of as the length of unimpeded habitat that would present an equivalent obstacle to the flow of a neutral allele down a uniform gradient.

from population 1 -perhaps to an external sink with still lower frequencies of this allele-while there is a steady inflow of the allele into population 2. These fluxes are such that the difference $\Delta u$ remains constant over time. This implies that the efflux from population $1,-\delta u$, must be balanced by an identical influx, $\delta u$, into the population. If all of this influx originates (albeit indirectly) from population 2, then the "effective migration rate", $m_{e}$, from 2 into 1 should be defined as:

$$
m_{e}=\delta u / \Delta u \text {. }
$$

$m_{e}$ can be calculated from the migration matrix $L$ which describes the movements between the complete set of interconnected populations. $L_{i j}$ $(i \neq j)$ is defined as the number of genes from $i$ which are found in $j$ after migration; $L_{i i}$ is defined as the (normalised) number of genes which remain in $i$, minus $1 . m_{e}$ is then found by solving the equation $\underline{u L}=\underline{\delta u}$, where $\underline{\delta u}$ is the row vector of fluxes into each population at equilibrium, with $\delta u_{1}>0, \delta u_{2}<0$, and $\delta u_{i}=0$ for $i \neq 1,2$. The vector $\underline{u}$ is the (normalised) number of genes in each population; $\Delta u=u_{2}-u_{1}$ (see equations (A1), (A6) for examples; this method is based on that of Bengtsson (1985), differing in that Bengtsson defined the matric $\underline{B}=\underline{L}+\underline{I}$, and derived $m_{e}$ from the leading eigenvector of $\underline{B}$ ). This effective migration rate seems a reasonable interpretation of what should be meant by the rate of gene flow from population 2 into population 1 .

This concept of an effective migration rate can also be used to describe the flow of genes between two populations which are separated by a genetic barrier. Within each population, we can consider each genetic background as a separate sub-population. The size of these sub-populations is maintained at an equilibrium by selection and migration, in the same way just as the sizes of demes are usually taken to be maintained by some sort of ciensity dependent regulation. Alleles can flow between different sub-populations in the same background by migration. The rate of increase of a neutral marker allele in population 1 due to migration from population 2 now depends not only on physical migration, but also on the frequency of each genetic background in population 1 and on how closely linked the marker allele is to any 
of the genes under selection. From a knowledge of the rate of increase of the neutral marker gene, $\delta u$, for a given difference $\Delta u$, it is again possible to define an "effective migration rate" between the two populations by using equation (2). It must now, however, be remembered that different marker loci will be associated with different effective migration rates.

Bengtsson (1985) introduced what he called the "gene flow factor" $\left(g f f=m_{e} / m\right)$, as a measure of the penetrability of a genetic barrier. In this paper we choose instead to talk about the strength of a genetical barrier between two populations. We define this strength as the inverse of the gene flow factor $\left(b=1 / g f f=m / m_{e}\right)$. When $b=1$, the effective migration rate is equal to the actual migration rate, and so there is no genetic barrier. On the other hand, when $b$ is large, the effective migration rate is much smaller than the actual migration rate, and so there is a strong barrier to gene flow between the two populations.

This definition of the strength of a genetic barrier, $b$, applies readily to one of the situations that we are going to consider: where there are two distinct populations which exchange migrants directly. The other situation that will be discussed is where two genetically different populations meet in a one-dimensional habitat, in which the density of the organism is everywhere constant. (The effects of varying density are discussed in Barton, 1986). The effect of a genetic barrier will then be studied by the way it influences the distribution over time and space of a selectively neutral allele. The frequency of the allele at position $y$ in generation $t$ is denoted by $u=u(y, t)$. As before, we assume that the allele frequency distribution is held at equilibrium by a continual influx of alleles into the right of the habitat, and by a continual efflux out of the left of the habitat. We also assume that the dynamics of the allele frequency distribution, away from the genetical barrier at position $x$, is governed by the diffusion equation:

$$
\frac{\partial u}{\partial t}=\frac{\sigma^{2}}{2} \frac{\partial^{2} u}{\partial y^{2}} .
$$

Here, $\sigma^{2}$ is the variance of parent-offspring distance (Nagylaki, 1975), and is taken to be the same everywhere.

As a measure of the strength of the barrier to the flow of genes from the right of the barrier across to the left, we will use the same definition as is given by Nagylaki (1976):

$$
B_{-}=\left(u_{x+}-u_{x_{-}}\right) /(\partial u / \partial y)_{x-}
$$

(Nagylaki's (1976) $\gamma$ is equal to $1 / B$ ). Here, $u_{x+}$ and $u_{x-}$ are the allele frequencies slightly to the right and to the left of the barrier at $x$, where the allele frequency changes abruptly (fig. 1(c)). The barrier to gene flow in the opposite direction is denoted by $\boldsymbol{B}_{+}$; when the barrier is symmetrical, the subscript can be dropped.

We can see from the following heuristic argument that this definition of barrier strength is analogous to the definition given above for two separate populations. Let population 1 consist of the individuals slightly to the left of $x$, and population 2 of the individuals slightly to the right of $x$. What was earlier called $\Delta u$ is now obviously $u_{x+}-$ $u_{x-}$. To find the flux into population 1 of the neutral allele, it is necessary to consider what happens to the left of this region. In this part of the species, the frequency of the neutral allele increases in every generation as a result of introgression across the barrier, by an amount $\int_{-\infty}^{x-}(\partial u / \partial t) d y$, which simplifies, by substitution from equation (3), to $\left(\sigma^{2} / 2\right)(\partial u / \partial y)_{x-}$. All these alleles must have come from the right of $x$, and all must have passed through population 1 . This value is therefore identical to the flux $\delta u$ in the expressions above. The strength of the barrier to gene flow from the right of $x$ to the left of $x$ can thus be rewritten using the earlier notation as $B_{-}=\left(\sigma^{2} / 2\right)(\Delta u / \delta u)$. Note, however, that $\delta u$ is here the integral of the rate of change of $u$ over space, and so has the dimension of distance per unit time; in the case of discrete demes, $\delta u$ has the dimension of per unit time.

The strength of the barrier to gene flow across a continuous population, $B$, has the dimension of distance. It can be thought of as the length of unimpeded habitat which would present an equivalent obstacle to the flow of a neutral allele (fig. 1(d)). The influence of the barrier on gene flow depends on the ratio, $(B / \sigma)$. If two populations which are fixed for alternative neutral alleles meet in a hybrid zone, introgression will be delayed for $\sim(B / \sigma)^{2}$ generations (from Barton, 1979). However, even slightly advantageous alleles will penetrate a localised barrier very rapidly (within $\sim \log \left((B / \sigma)^{2} \pi s / 2\right) / 2 s$ generations if $s \gg$ $\left.(B / \sigma)^{-2}\right)$. Such alleles will be delayed for much less time than they would need to cross a length $B$ of unimpeded habitat: although the effect of physical distance and of a localised barrier on a neutral allele are equivalent, simple physical distance is a much more effective obstacle to the spread of advantageous alleles than a localised genetical barrier.

The effect of a barrier between two discrete populations is similar. Here, the barrier strength, $b$, is dimensionless. Neutral alleles will be delayed 
for $\simeq b / m$ generations, whereas slightly advantageous $\left(s>(b / m)^{-1}\right)$ alleles will be delayed for $\approx \log ((b / m) s) / s$ generations. We can see that although the barrier strengths $B$ and $b$ are analogous, in that they are both proportional to the ratio between gene frequency difference and flux $(\Delta u / \delta u)$, the ratios $(B / \sigma)^{2}$ and $(b / m)$ are the parameters which determine the effect of a localised barrier on genetical divergence.

\section{RESULTS}

We have derived a series of expressions for the strength of the barrier to gene exchange between either two discrete demes, or across a continuous habitat. These derivations are given in the Appendix. We will now consider the effects of various factors: the strength of selection, the position of the marker locus, the number of selected loci, and epistatic interactions between loci. Since we cannot consider more than a tiny proportion of combinations of these factors, we largely restrict attention to the dependence of barrier strength on each factor separately in the limiting cases where selection is either very strong, or very weak, relative to recombination. Throughout, it should be remembered that the parameters $(b / m)$ and $(B / \sigma)^{2}$, which have the dimension of time, must be large $\left(\geq 10^{4}\right.$ generations, say) to retard the spread of even a neutral allele for an evolutionarily significant period.

\section{Strength of selection}

The strength of a genetic barrier to the flow of an allele into a new population is determined by the reiationship between the strength of the selection directed against the various possible hybrid genotypes, and the closeness with which the marker locus is linked to the genes selected against in the new population. We start our analysis by investigating this relationship for the "rod model" (fig. 2(a)), where equations (A3)-(A5) give us results which are as exact as possible. We assume that selection acts independently against introgressing alleles at each of the $n$ loci, so that the fitness of an individual with $i$ foreign alleles is $v_{i}=(1-s)^{i}$. As a measure of the total strength of selection on the barrier, we will use $S=n s$; the ratio between $S$ and the total map length (defined as $R=n r$ ) is denoted by $\theta$.

The strength of the barrier to a marker allele at the end of a selected "rod" chromosome is plotted in fig. 3(a), for various $S$ and $R$. Here, the (a)

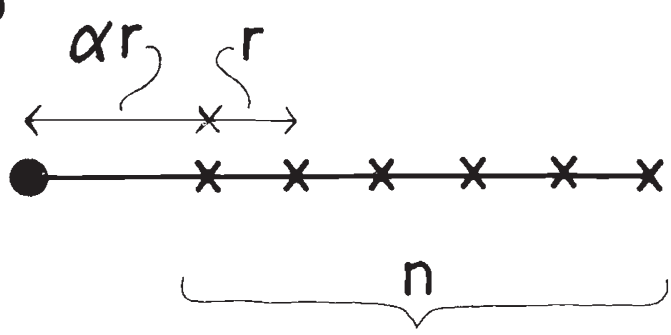

(b)

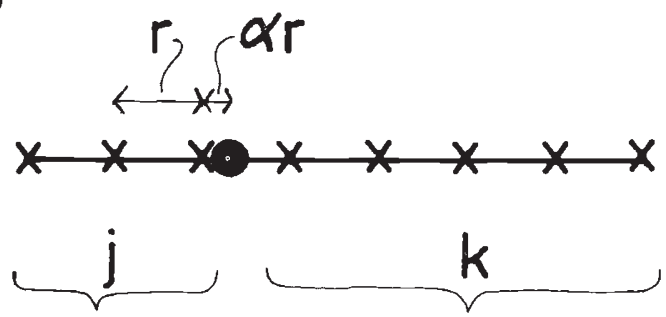

Figure 2 (a) The rod model. A marker gene (solid circle) lies at the end of a chromosome which carries $n$ selected genes. The rate of recombination between the marker and the nearest selected gene is $\alpha r$ whilst the recombination rate between adjacent selected genes is $r$. (b) A marker locus embedded in a selected chromosome. $j$ selected loci lie to the left, and $k$ to the right. The recombination rates between the marker and its two neighbours are $\alpha r$ and $(1-\alpha) r$

distance between the marker locus and the first selected gene is the same as the distance between the different selected genes (i.e., $\alpha=1$ ). The map length from the marker locus to the most distant selected locus is thus exactly $R$. From the figure, it is seen that most of the variation in barrier strength can be explained by the ratio $\theta=S / R$, especially when $S$ is small: for $S<0.1$ (provided $\theta$ is less than 10), the difference between the exact result (equation (A4)) and the one estimated by equation (A3) is at most a few per cent. There is, in any case, good biological reason to assume that $S$ should not be particularly large. As discussed above, our analysis is here centered on the importance of genetic factors linked to the marker locus. There may well be other genes involved in producing the barrier, but if they are not linked to the chromosome carrying the marker, the total barrier strength will simply be the product of effects of the two classes of loci (see Bengtsson, 1985).

In the remainder of the analysis, we will therefore consider only small $S$, in the expectation that the results for stronger selection would be qualitatively similar. We have only shown that this is so for the "rod model" in two demes. The mathematical similarity between rod and embedded models 


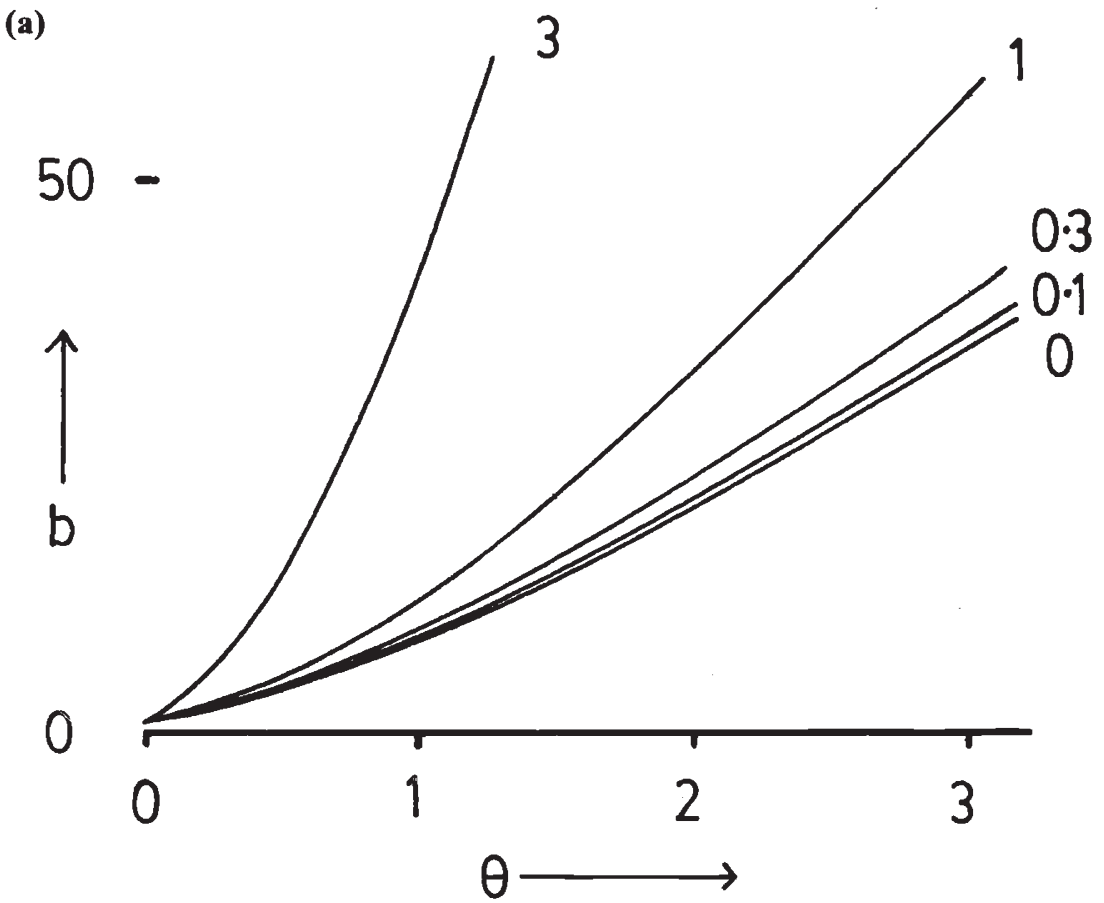
Figure 3(a) The barrier to gene flow $(b)$ at a locus at the end of a selected chromosome, plotted against $\theta=S / R$, for $S=0,0 \cdot 1$,
$0 \cdot 3,1$, and $3 . n=20, \alpha=1$.

(b)

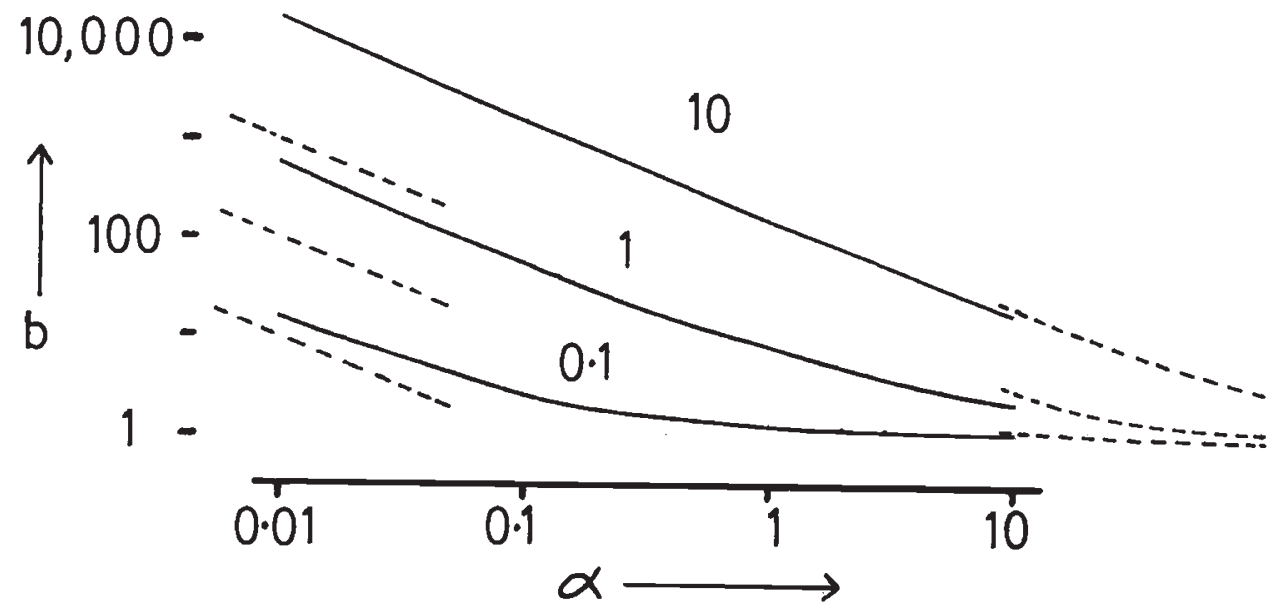

Figure 3(b) The barrier strength $(b)$ as a function of the position of the marker locus, in the rod model (fig. 2(a)). Values are for the limit of weak selection (equation (A5)), and for $\theta=0 \cdot 1,1,10 . n=20$. The left-hand dotted curves show the asymptotic relation $b=(\theta / \alpha)$, and the right-hand curves, $b=1+n \theta / \alpha$.

(compares equations (A1), (A6) and see below) suggests that strong selection will not give qualitatively new results for the latter case when two discrete demes are considered. In a continuous habitat, the diffusion approximation on which all our results are based breaks down when selection is strong. However, if the organisms are in fact distributed into a chain of demes, arranged like stepping-stones, our results for discrete demes will be applicable when selection becomes so strong that most gene frequency changes occur between two adjacent demes. 


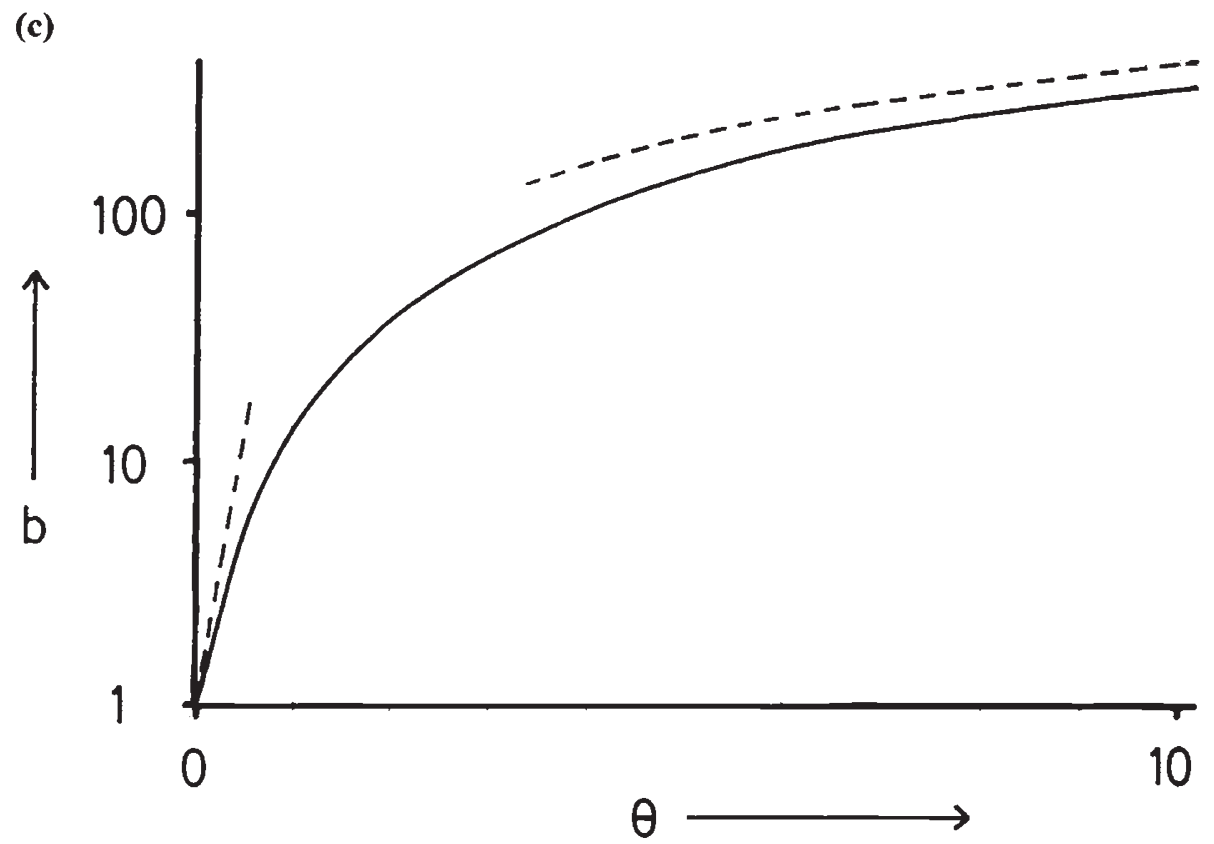

Figure 3(c) The barrier strength ( $b$; solid curve), compared with the asymptotic relations (dotted curves), $n^{\theta} e^{1.96 \theta}(\theta$ small), and $2 n \theta\left(\theta\right.$ large), in the limit of weak selection (equation (9)). $\alpha=\frac{1}{2}, n=20$.

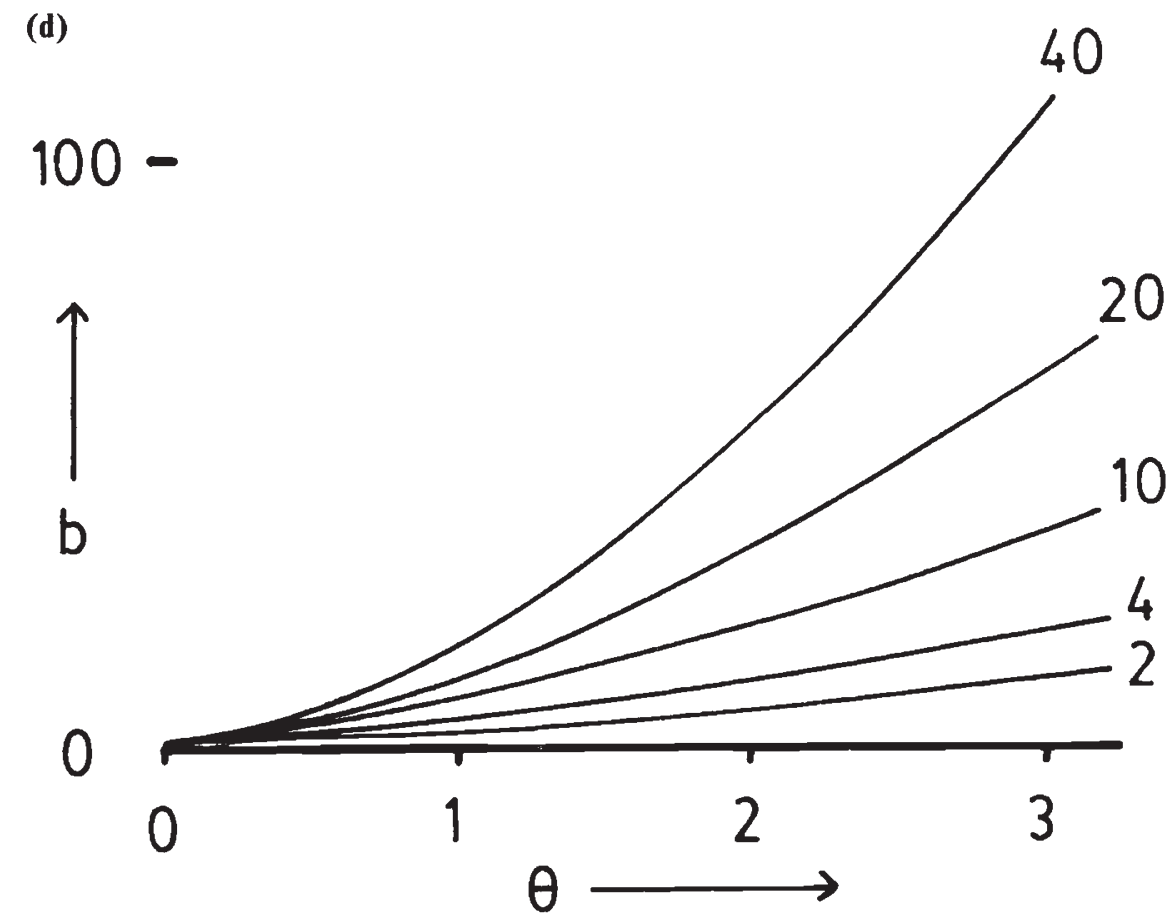

Figure 3(d) As in fig. 3(c), but with $n=2,4,10,20,40$. 


\section{Position of the marker locus}

We begin by considering the rod model, in which the marker locus lies at the end of a selected chromosome. The barrier strength is plotted against $\alpha$ for various values of $\theta$ in fig. 3(b); $n=20$. The relation between the position of the marker and the barrier strength can be understood by taking limits of equation (A5) with respect to $\alpha$.

When the marker is far from the block of selected genes $(\alpha \gg n)$, the block acts as a single unit; the barrier strength becomes close to $1+(n \theta / \alpha)$, which is the same as would be produced by a single gene with effect $S$, a distance $\alpha r$ map units from the marker (Bengtsson, 1985). At the other extreme, when the marker locus is very close to one of the selected loci $(\alpha \ll 1)$, the barrier becomes very strong. However, although the barrier strength becomes proportional to the ratio of selection to recombination $(s / \alpha r=\theta / \alpha)$, as would be expected from the effect of the single closest locus, the residual effect of all the other genes reduces gene flow substantially more than would be expected from the single locus result (fig. 3(b)).

It is most interesting to look at a case somewhere between these two extremes. We will now concentrate on $\alpha=\frac{1}{2}$; this can later be compared with the more realistic case, where a marker locus is embedded halfway between the two closest selected loci. When selection is much stronger than recombination $(S \gg R)$, the chance that two recombination events will occur within the region before the block of deleterious introgressing alleles is eliminated is exceedingly small. We therefore only need to consider the chance that the marker gene will recombine away from the selected block in one step. The chance per generation of such a recombination is $(r / 2)$, whilst the chance per generation of selective elimination is $S=n s$. The barrier strength is therefore $b=2 n s / r=2 n \theta$. Thus, for a strong barrier, the rate of gene flow is reduced by a factor equal to the ratio between the total selection against $F .1$ hybrids, and the recombination rate with the nearest selected locus.

At the other extreme, when $S \ll R$, we need only consider the pairwise interactions between each selected locus and the marker: since selection is relatively weak, interactions between the selected loci can be neglected. (This is equivalent to discarding higher order disequilibria, as was done in the Appendix for continuous populations.) We consider the barrier generated by a fixed selection pressure, acting on a chromosome of fixed map length, and spread over $n$ loci. The effect of a single locus of effect $s$ at a map distance $r$ is $b=(1+(s / r))$ when $s, r \ll 1$ (from Bengtsson, 1985, equation (1)); when $\theta \ll 1$, this is approximately $\exp (s / r)$. Hence, the net barrier strength is approximately $\exp \left(\sum_{j}\left(s / r_{j}\right)\right)=\exp (S / \bar{r})$, where $r_{j}$ is the recombination rate between the marker and the $j$ 'th locus, and $\bar{r}$ is the harmonic mean of the $r_{j}$. For the rod model with $\alpha=\frac{1}{2}$, the limit of this harmonic series for large $n$ gives $b=n^{\theta} e^{1 \cdot 96 \theta}$; the same result can be obtained by taking the limit of equation (A5) for small $\theta$. The relation between $b$ and $\theta$ is plotted in fig. 3(c) for $n=20$ loci; the asymptotic forms for both large and small $\theta$ agree well.

Now, consider a marker locus embedded symmetrically in the centre of a selected chromosome (Fig. 2(b); $\alpha=\frac{1}{2}, j=k=n / 2$ ). When selection is much stronger than recombination, the selected alleles tend to remain together in a strongly selected block; the frequency of such blocks is $(m / S)$, where $m$ is the migration rate into the population. The rate of production of blocks which have been broken next to the neutral locus is $r(m / S)$; this will be balanced by selection against half-blocks to give an equilibrium frequency of $(m / S) r /(S / 2)$. Finally, free neutral alleles will be recombined away from these blocks at a rate $(r / 2)(m / S) r /$ $(S / 2)=m(r / S)^{2}$. Hence, the barrier strength is approximately $(n \theta)^{2}$ when $\theta \gg 1$. This can be confirmed by direct solution of equation (A6).

When selection is relatively weak $(\theta \ll 1)$, the barrier strength can be calculated from the harmonic mean recombination rate, as before; it is approximately $n^{2 \theta} e^{2 \cdot 5 \theta}$. Thus, the barrier at the centre of a selected chromosome is roughly the square of the barrier at the edge, for both large and small $\theta$ (compare figs. 3(d), 4(a)). This is because two recombination events are needed to transfer the marker allele to the new background, rather than one. However, as long as the marker locus is not very close to the end of the chromosome, the barrier strength does not vary by very large factors as position changes. The average barrier to flow at marker loci evenly distributed along the chromosome (but always halfway between the adjacent selected loci: $\alpha=\frac{1}{2}$ ) is, by considering the limiting solutions of equation (A6), $\frac{2}{3}$ of the maximum barrier at the centre when $\theta \gg 1$, and roughly equal to the maximum when $\theta \ll 1$ and $n \gg 1$ (fig. 4(b)). To put this another way, 70 per cent of the chromosome is within a factor of 2 of the maximum barrier strength, even when $\theta$ is large; the proportion tends to 100 per cent for small $\theta$.

The dependence of barrier strength on chromosomal position is essentially the same in the case 


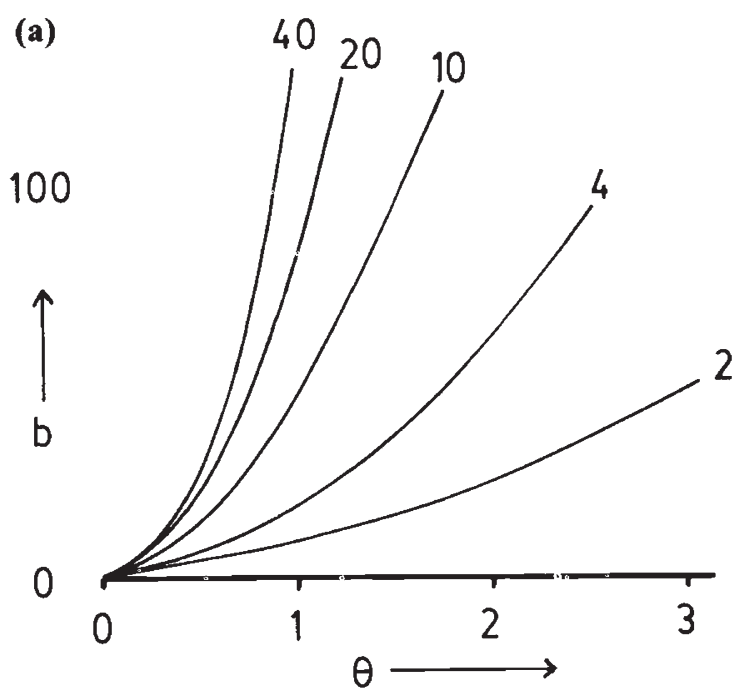

Figure 4(a) The barrier strength (b) for a locus embedded symmetrically in the centre of a selected chromosome $(\alpha=$ $\left.\frac{1}{2}, j=k=n / 2\right)$, plotted against $\theta=S / R$, in the limit of weak selection. $n=2,4,10,20,40$ (cf. fig. 3 (c)).

(b)
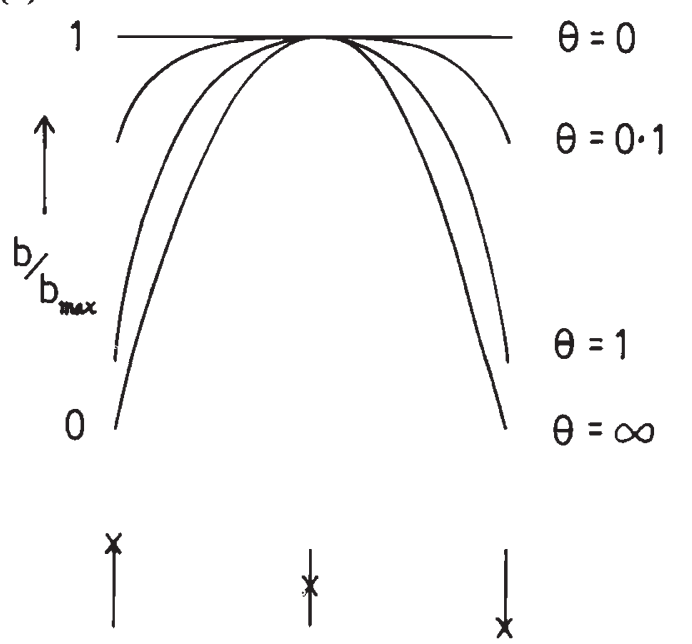

Figure 4(b) The barrier strength at different positions along the chromosome, relative to the strength at the centre. $\alpha=\frac{1}{2}$ throughout. The pattern does not depend much on the number of loci; here, $n=20$.

of a continuous population. When selection is much stronger than recombination, the barrier strength was derived by using the same argument as has been given above for a discrete deme, and so may be expected to behave in the same way. Since the proportion of singly recombinant chromosomes, $z$, is proportional to $(r / S)$ (equation (A9)), and since, by dimensional arguments, such recombinants are found over a region of width proportional to $\sigma / \sqrt{S}$, the integral in equation
(A11) is proportional to $r^{2} /\left(\sigma \sqrt{ } S^{3}\right)$. Hence, $(B / \sigma)$ is proportional to $\sqrt{S^{3} / r^{2}}=n^{2} \theta^{2} / \sqrt{S}$ : the dependence on $n$ and $\theta$ for fixed $S$ is the same as for discrete demes. When selection is much weaker than recombination, the barrier strength has again been derived in the same way as for a discrete deme, and in both cases depends only on the harmonic mean recombination rate. Since the harmonic mean is dominated by relatively loosely linked loci when $n$ is large, it is only weakly dependent on the position of the marker locus (fig. 4(b), $\theta=0 \cdot 1)$. Thus, in this case barrier strength becomes more or less independent of marker position, regardless of population structure.

\section{Number of selected genes}

In all the models we have considered, the strength of the barrier has increased as the overall selection pressure $(S)$ has been spread over a larger number of loci, within a genetic map of fixed length. One reason for this is that as the selected loci increase in numbers, the marker locus tends to become more closely linked to them. When selection is strong relative to recombination, the barrier strength is proportional to the product of the rates of recombination with the selected loci on either side. Since these rates are each inversely proportional to the number of loci, for a fixed total map length, the barrier strength is proportional to $n^{2}$; this is true for both a discrete deme and for a continuous habitat. When selection is relatively weak, the barrier strength depends on the harmonic mean recombination rate, $\vec{r}$, and is proportional to $\exp (S / \bar{r})$ for both a discrete deme (from equation (A5)), and for a continuous population (from equation (A21)). Since the sum of a harmonic series diverges logarithmically $(1+(1 / 2) \cdots$ $(1 /(n-1) \sim \log (n))$. The harmonic mean is approximately $R /(2 \log (n))$ when the marker is embedded in the chromosome, and when $n$ is large. The barrier strength is therefore proportional to $n^{2 \theta}$ (figs. 3(c), 3(d), 4(a)).

In continuous populations, there is another reason why the strength of the barrier to gene flow increases with the number of loci under selection. The barrier has the dimensions of a distance, and is proportional to the width of the clines at the selected loci (from equation (A16)). When selection is strong enough that most chromosomes carry intact blocks of genes from the populations on either side, the cline width depends on the total selection against heterozygotes, and not on the number of loci (equations (A8)). However, when selection is relatively weak, the cline width 
depends on the selection pressure on individual loci. This decreases with the total number of loci $(s=S / n)$. So, the cline width, and the barrier strength, are both proportional to $\sigma / \sqrt{s}$, and hence to $\sqrt{n}$ (equations (A21), (A25)).

\section{Selection/recombination}

The key parameter determining the strength of the barrier, in all models, is the ratio between selection and recombination. The dependence of gene flow on this ratio is very similar to the dependence of the equilibrium frequency of introgressing deleterious alleles (Barton, 1983). In that case, in the limit where very many genes are involved $(\log (n) \gg 1)$, there was a sharp transition between two qualitatively different regimes when $\theta$ passed a certain critical value. In the case of flow between discrete demes, when $\theta>1$, selection could act strongly enough to maintain a definite equilibrium frequency even when an infinite number of loci was involved. However, when $\theta<1$, selection could not prevent the increase of the introgressing alleles: recombination then breaks up the blocks of introgressing genes into smaller and smaller segments, which are selected against less and less strongly. This type of result is not obtained when the strength of the barrier to neutral alleles is studied. Although the rate of gene flow does depend strongly on the ratio between selection and recombination, there is no critical value at which the barrier strength suddenly becomes much stronger. In the limit where an infinite number of genes are involved, the barrier simply becomes infinitely strong.

One can, however, understand the threshold behaviour of the frequency of selected alleles by using the results on gene flow derived here. Consider the frequency of a selected allele as being maintained by a balance between its own (relatively weak) selective effect $(s=S / n)$ and the effective rate of immigration, which will be determined largely by selection on all the linked loci. When $\theta$ is small, this effective immigration rate is proportional to $n^{-2 \theta}$; since the counterbalancing selection pressure is inversely proportional to $n$, we may expect the equilibrium frequency to be proportional to $n^{-2 \theta+1}$. As $n$ tends to infinity, this tends to zero if $\theta>\frac{1}{2}$, but to infinity if $\theta<\frac{1}{2}$. An exact calculation of the threshold, valid for large $\theta$, is more complicated (Barton, 1983), but this heuristic argument explains why the frequency of the selected genes changes sharply at a critical value of $\theta=S / R$, whereas the barrier strength increases smoothly with $S / R$ even when $n$ is large.

\section{Epistasis}

Thus far, we have assumed that selection acts independently (i.e., multiplicatively) against heterozygotes at each locus. This may be a reasonable model of certain types of multiple chromosome rearrangement, but seems unlikely to be generally realistic. Here, we generalise the model of selection by supposing that the fitness of an individual with a proportion $h$ of heterozygous loci is $\exp (-\operatorname{Sh}(\gamma+1(1-\gamma) h))$. When $\gamma=1$, this gives essentially the same model as was used above. When $\gamma<1$, selection acts more strongly against heterozygotes when the proportion of heterozygous loci is high, whilst when $\gamma>1$, selection acts more strongly when the proportion of heterozygotes is low (fig. 5). Since overall fitness will only decrease monotonically with heterozygosity if $0<$ $\gamma<2$, we only consider this range of $\gamma$.

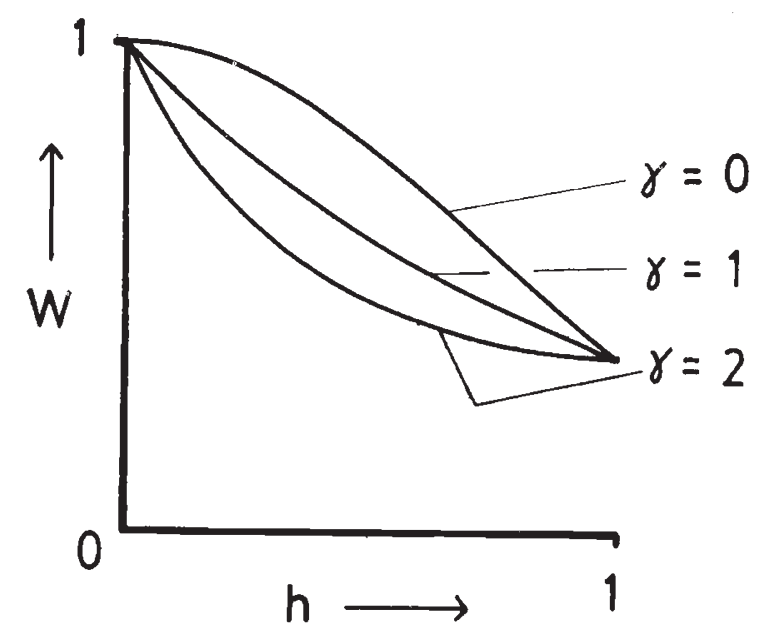

Figure 5 The relation between individual fitness, $W$, and the proportion of heterozygous loci in an individual, $h: W=$ $\exp (-S h(\gamma+(1-\gamma) h))$; here, $S=1$.

The relation between the degree of epistasis $(\gamma)$ and the barrier strength $(b)$ is plotted in fig. 6 (a) for various values of $\theta=S / R$. These calculations are made for a marker at the end of a selected chromosome (the rod model), and are exact: they do not require the assumption that selection is weak. The barrier strength always increases with $\gamma$; this is because the fitness of introgressing genotypes decreases with $\gamma$ for a fixed fitness of F1 hybrids (fig. 6). When selection is weak relative to recombination $(\theta=0 \cdot 1)$, barrier strength decreases by a factor of $2 \cdot 1$ as $\gamma$ decreases from 2 to 0 . However, when selection is relatively strong $(\theta=10)$, the barrier strength decreases by a factor 
of 2.4 as $\gamma$ decreases from 2 to 0.25 , but then drops dramatically, by a factor of $5 \cdot 9$, as $\gamma$ decreases to 0 (fig. 6(a)). This is because when selection is relatively strong, most gene flow occurs through a single recombination, which immediately transfers the marker gene from the F1 type heterozygote

(a)

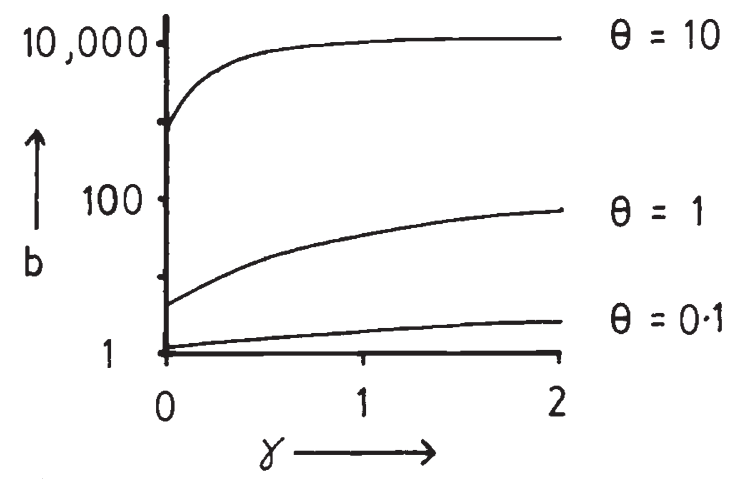

(b)

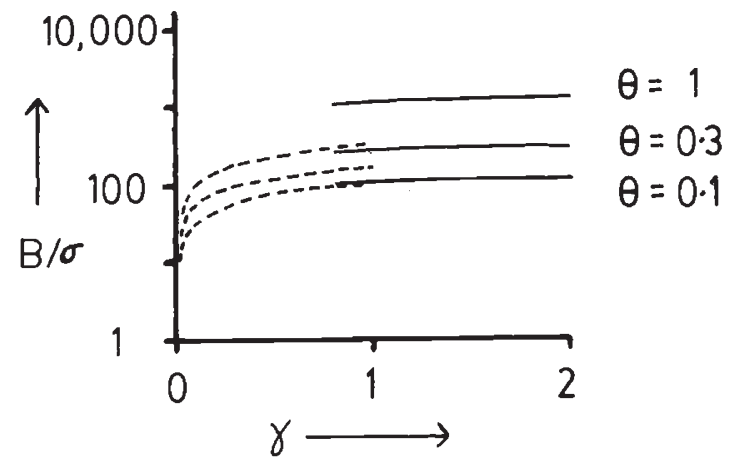

Figure 6 (a) The relation between barrier strength $(b)$ and epistasis for the rod model (eq. A3). Here, $R=0.3, \alpha=\frac{1}{2}$, and $n=100 . S=0.03,0 \cdot 3$, and 3, corresponding to $\theta=0 \cdot 1$, 1,10 . (b) The relation between barrier strength (relative to dispersal distance: $\boldsymbol{B} / \sigma$ ) and epistasis in a continuous habitat, where selection is weak relative to recombination. The marker locus is embedded symmetrically in the centre of a selected chromosome, of map length $R=0 \cdot 3$, and carrying $n=100$ selected loci $\left(\alpha=\frac{1}{2}, j=k=50\right)$. When $\gamma>1$, the clines at different loci will be attracted to each other, and will coincide (solid curve). When $\gamma<1$, the clines will repel each other, and the solution in which all clines coincide may become unstable. The barrier strength will then be the sum of contributions from clines lying at different places (dotted curve). Values are calculated using equations (A16), (A20). (c) The relation between barrier strength (relative to dispersal distance: $B / \sigma$ ) and epistasis in a continuous habitat, where the total selection pressure is strong relative to recombination $(\theta=10)$. Other parameters are as above. For the case where all clines coincide (solid curve), values are calculated using equations (A9)(A10). When the clines lie at different positions (dotted curve), the selection acting on each individual locus is weak $(s=\gamma S / 100<0.03)$, and so values are calculated using equations (A16), (A20).

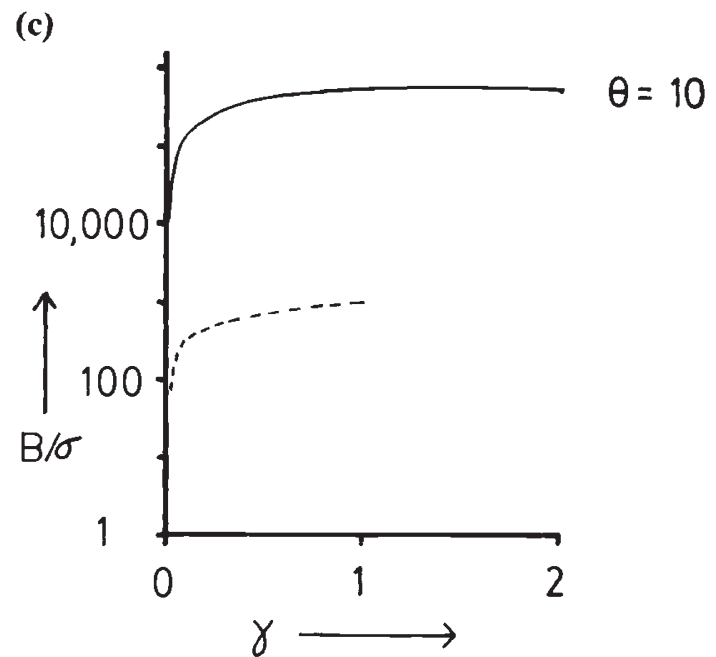

Figure 6 (cont.)

into the native genotype. The rate of flow therefore depends primarily on the frequency of F1 type hybrids; since their fitness is set at $e^{-S}$, this rate would not be expected to depend much on $\gamma$. However, when $\gamma$ becomes small (here, $<0.25$ ), this approximation breaks down: the selection pressure against genotypes carrying only a few introgressing genes becomes small, and so these genotypes increase to a relatively high frequency. They will then make a substantial contribution to gene flow, and the barrier will become much weaker.

We should stress that the above discussion of gene flow into discrete demes rests on the assumption that the actual rate of immigration $(m)$ is small enough, compared with selection, that hybrids are rare. When immigration becomes high relative to selection, this assumption breaks down; above some threshold, all differentiation may be lost (Karlin and McGregor, 1972). Thus, as $\gamma$ becomes very small, chromosomes carrying only a few foreign alleles may be at such a small selective disadvantage that the demes are no longer genetically distinct, and the barrier collapses entirely.

The effects of epistasis on gene flow across a continuous habitat are similar. Consider, first, the case where selection is weaker than recombination. Then, the barrier to gene flow depends on the spatial pattern of mean fitness, $\bar{W}(x)$ (equations (A15), (A16)). We will therefore examine the effects of epistasis by keeping the mean fitness of the population at the centre $(\vec{W}(0))$ fixed, rather than by keeping the fitness of F1 heterozygotes fixed, as above. This seems reasonable, because in a continuous population, F1 type hybrids will be 
extremely rare when the number of loci is large and selection is not extremely strong, whereas the mean fitness of a natural population is, at least in principle, measurable. The relation between mean fitness, and the shape of the cline at the marker locus, is illustrated on fig. 7 , for $\gamma=1$. Although the gradient at the marker locus is proportional to $\bar{W}^{(-1 / \tilde{r})}$ (equation (A15)), this does not imply that the barrier strength depends only on the depression in mean fitness at the centre of the cline (which we here keep fixed, and independent of $\gamma$ ). The barrier strength also depends on the width of the region, over which fitness is reduced, which depends in turn on the relation between mean fitness and genotype frequencies (equation (A20)).

The relation between barrier strength and epistasis is plotted in the solid curves in fig. 6(b), for various values of $\theta=S / R$. (The upper curve, for $\theta=1$, should perhaps not be taken too seriously, since the derivations assume that $\theta$ is small.) Although, as expected, larger values of $\gamma$ give stronger selection against hybrids, and hence a stronger barrier to gene flow, the effects of varying epistasis are not great, and are of the same order as for discrete demes. A complication arises, however, when we consider $\gamma<1$. Then, heterozy- gosity at one locus causes a greater reduction in fitness when heterozygosity at other loci is higher. This form of epistasis will destabilise the symmetrical solution in which all clines coincide: the clines at different loci will tend to move apart and will come to lie in different places. Although linkage disequilibrium will counteract this separation, we expect that when selection is weak relative to recombination, the coincident solution will become unstable when $\gamma$ is smaller than some critical value (cf. Slatkin, 1975). In the dotted curve in fig. 6(b), the barrier caused by $n$ clines lying in different places is plotted against $\gamma$. When $\gamma=1$, the barrier produced by a set of staggered clines has roughly the same strength as one produced when the clines coincide, provided that $\theta$ is small (cf. Barton and Hewitt, 1983, fig. 2). However, as selection becomes stronger, interactions between loci strengthen the barrier when the clines coincide. When $\gamma$ is small, the selection against heterozygotes at a single locus is also small $(\gamma S / n)$, and so the barrier to gene flow tends to zero.

The dependence of the barrier on epistasis when selection is relatively strong $(\theta=10)$ is plotted in fig. 6(c), using the approximation of equations (A9)-(A11). In this case, strong linkage

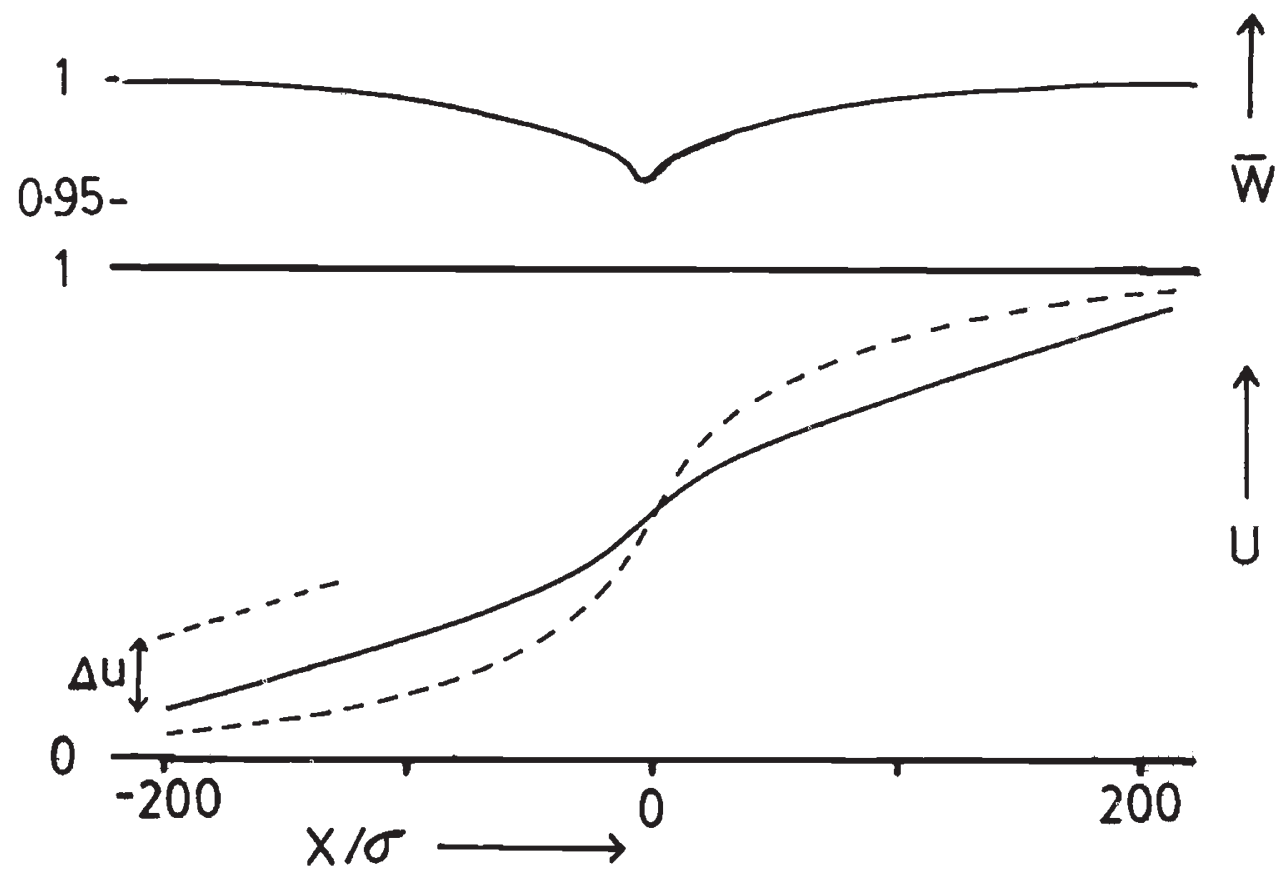

Figure 7 The barrier to gene flow produced by a total selection pressure $S=0.09$, acting against heterozygotes at $n=100$ loci. The marker locus is embedded symmetrically at the centre of a chromosome of map length $R=0 \cdot 3 ;$ thus, $\theta=S / R=0 \cdot 3$. This selection maintains clines at the selected loci (dotted curve). The mean fitness at the centre is reduced to $e^{-S / 2}=0 \cdot 956$ (upper curve). Linkage disequilibrium between the selected genes and the marker produces a step $\Delta u$ in the cline at the marker locus (lower solid curve). The strength of the barrier to gene flow is $B=232.5$ dispersal distances (from equation (A22)). 
disequilibrium may keep the clines in the same place even when $\gamma$ is small; we have therefore plotted the solid curve, for the coincident solution, down to smaller $\gamma$. The pattern is then close to that seen in the case of flow into a discrete deme: the barrier strength depends little on epistasis when $\gamma>0.25$, but collapses when $\gamma$ becomes small. The explanation for this collapse is the same as for a discrete deme: as $\gamma$ approaches zero, the frequency of recombinant chromosomes increases, allowing free gene flow. This may seem surprising in this case, because in the approximation used to calculate fig. $6(\mathrm{c})$, the rate of gene flow is proportional to the frequency of recombinants of the type $+++\cdot---;$ these increase indefinitely as $\gamma$ tends to 0 , even though such chromosomes are usually found in individuals with half their loci heterozygous, and whose fitness we keep at a fixed value relative to the parental genotypes. This increase of recombinant chromosomes occurs because at the centre of the cline, average heterozygosity is one half, and so these recombinants are not at a relative disadvantage. Since they are continually produced by recombination, they may rise to a high frequency (cf. similar consequences of spatially varying selection coefficients in Nagylaki, 1975). The lower dotted curve in fig. 7 shows the barrier strength produced when all the clines lie in different positions. In this case, each cline involves selection $\gamma S / n$ at a single locus; the barrier can be calculated assuming that $(\gamma S / n) \ll R$, from equations (A16), (A20). The barrier produced by a set of staggered clines is now much weaker than when the clines coincide, as would be expected by extrapolation from fig. 6(b).

We can, therefore, summarise the effects of this model of epistasis by saying that the barrier to gene flow does not depend greatly on the precise relation between fitness and heterozygosity unless selection is strong relative to recombination, and the deleterious effects of a few heterozygous loci are much smaller than would be expected from the fitness of F1 hybrids, or of populations at the centre of a hybrid zone. However, we have only considered one of a very wide range of possible selection schemes. In particular, we have assumed that selection acts against heterozygotes. Suppose instead that selection acts against recombinant genotypes. In the case of flow into an isolated deme, the F1 type hybrids would reach a much higher frequency, since they would not be selected against. When selection is strong relative to recombination, this might greatly weaken the barrier, since gene flow then occurs mainly through two recombination events, and its rate is proportional to the frequency of F1 type hybrids (see above). However, we stress that when selection is weaker than recombination, the effect may be much smaller: here, the barrier is due to the cumulative effects of each individual selected locus, and so depends only on the selection pressure against each individual gene when set against the native background. Similar arguments apply in continuous habitats. When selection is much stronger than recombination, the rate of flow depends on the frequency of F1 type heterozygotes, which may be much higher if these are not at a selective disadvantage. However, when selection is weak, the rate of flow depends only on the spatial pattern of mean fitness $(\bar{W}(x))$, and not on whether mean fitness is reduced by recombination rather than by heterozygosity.

\section{DISCUSSION}

We, and others, have earlier described and discussed the effect of genetical barriers on gene flow between differentiated populations (Bengtsson, 1974; Barton, 1979; Spirito et al., 1983; Petry, 1983). These previous results dealt primarily with the effects of genetic divergence at one or a few loci. In the present article, we have extended the analysis to the effects of divergence at large numbers of loci. The conclusions fit with those expected by extrapolation from the single locus case: in order for gene flow to be significantly reduced over much of the genome, hybrids must be substantially less fit, and the number of genes involved in building the barrier must be so large that the majority of other genes become closely linked to some locus which is under selection. This implies that populations must diverge considerably before they can be reproductively isolated despite being in parapatric contact via a hybrid zone.

It is easy to believe that because two populations can be shown to exchange genetic material, they must therefore rapidly become similar. This is, of course, not necessarily true; the outcome will depend crucially on the selective effects of the genes in question. If two differentiated populations come into secondary contact after a period of separation, some genes may have diverged in such a way that selection will maintain different alleles in two different environments or genetic backgrounds. Such selectively maintained divergence can persist indefinitely, and will-as described above-play a part in reducing the rate of gene flow at other loci. Neutral alleles will introgress, but the process of mixing will take a long time, 
simply because the random diffusion of genes through a spatially distributed population is slow process. Imagine, for example, that the populations have been in contact for 10,000 years, and that the dispersal range is 800 metres in a year. Then the effect of gene flow between the two populations will be perciptible $50 \mathrm{~km}$ from the contact zone, but almost no evidence of the contact will be found $100 \mathrm{~km}$ from the zone (an example relevant to the karyotypic races of Sorex araneus in Sweden; see Frykman, 1983). It is thus wrong to expect an effect of gene flow in all parts of an organism's distribution, just as it would be wrong to believe the converse, that if two parapatrically distributed taxa differ in allele frequencies at some localities, they cannot therefore be in genetic contact.

Two hybridising populations will, however, evolve in consort, in that an advantageous mutation which occurs in one population will be able to pass through even a strong genetical barrier, and then continue to spread into the other population. From this point of view it is reasonable to continue to refer to populations which are subdivided by clear hybrid zones as belonging to the same biological species (see Barton and Hewitt, 1985).

The conclusion that strong selection against hybrids at numerous genetic loc. is required to give a strong barrier suggests to us that reproductive isolation is likely to evolve slowly and in a large number of steps. Certainly, it is hard to see how the genetic changes needed for substantial isolation could arise during one or a few drastic "founder events" or "genetic revolutions". More generally, one can argue that a strong barrier to gene flow cannot exist, and persist, unless the two populations involved have come to rest at alternative "adaptive peaks" which are separated by a deep fitness trough. It seems highly unlikely in that case that drift could have caused the populations to have evolved into such different states in only a few steps (Walsh, 1982; Barton and Charlesworth, 1984).

Models have been proposed in which strong selection against F1 and F2 hybrids is achieved, even though the selection acting during the evolutionary divergence is weak. The simplest example is a two locus model proposed by Dobzhansky (1937), in which selection acts against double heterozygotes, but not against the initial substitution of alleles at one or other locus (see Bengtsson and Christiansen, 1983, and analogous models by Nei et al., 1983; Wills, 1970; and Lande, 1981; the latter two publications discuss premating, rather than postmating, isolation). At first sight the existence of such models seems to contradict the above claim that drift cannot cause population to evolve into states where they are differentiated by a deep fitness trough. Furthermore, Bengtsson (1985) has shown that the Dobzhansky model will lead to genetic barriers which may be of considerable stiength. However, this is only the initial situation. Persistent flow between the differentiated populations will rapidly change them in such a way that the genetic barrier becomes weakened. This effect becomes particularly clear in models of a continuous habitat, where the contact between the different types is direct and immediate for organisms close to the hybrid zone. The effect on gene flow in a multilocus generalisation of the Dobzhansky model has been analysed above in the case of epistasis, with small $\gamma$. Here, although the mean fitness of the hybrid population formed after initial contact is fixed at a low and perhaps small value, the barrier to gene flow collapses as $\gamma$ decreases (see figs. 6(b) and 6(c)). In other words, the fitness of the initial hybrid population, or for that matter of the F1 and F2 hybrids, does not reflect the eventual effect on gene flow; the Dobzhansky model, and similar schemes, would not in fact produce much reproductive isolation.

We can illustrate the collapse of a potentially strong barrier to gene flow, by reference to the contact zones between the karyotypic races of the common shrew, Sorex araneus, where different populations carry different metacentric chromosome arm configurations (Searle, 1984; Fredga, 1986). Hybrids between such different karyotypes have severe meiotic difficulties, and hence substantially reduced fertility. However, there is a clear local increase in acrocentric chromosomes within hybrid populations, which greatly reduces the expected infertility. The differences between the chromosomal races may well have evolved by drift, and can, in principle, lead to a strong genetical barrier. However, in practice this barrier disappears when the different karyotypic forms come together. Genetic differences of this sort can only act as a stable barrier to gene flow if all genetic variation of the ancestral type (in this case, the acrocentric chromosomes) has been lost from the hybridising populations.

Acknowledgements We are grateful for the support this work received from grants from the Nuffield Foundation and the Science and Engineering Research Council (to the first author), and from the Swedish Natural Science Research Council (to the second author). We would also like to thank Penny Akam for her patient typing. 


\section{REFERENCES}

Barton, N. H. 1979. Gene flow past a cline. Heredity, 43, 333-339.

BARTON, N. H. 1983. Multilocus clines. Evolution, 37, 454-471.

BARTON, N. H. 1986. The effects of linkage and density-dependent regulation on gene flow. Heredity, 57, 415-426.

BARTON, N. H. AND CHARLESWORTH, B. 1984. Genetic revolutions, founder effects, and speciation. Ann. Rev. Ecol. Syst., 15, 133-164.

BARTON, N. H. AND HEWITT, G. M. 1983. Hybrid zones and speciation. In: Evolution and Speciation: essays in honour of M. J. D. White, Atchley, W. R. and Woodruff, D. S. (eds.) Cambridge, Cambridge University Press, pp. 109145.

BARTON, N. H. AND HEWITT, G. M. 1985. Analysis of hybrid zones. Ann. Rev. Ecol. Syst., 16, 113-148.

BAZYKIN, A. D. 1969. Hypothetical mechanism of speciation. Evolution, 23, 685-687.

BENGTSSON, B. O. 1974. Karyotype evolution in vivo and in vitro. D. Phil. thesis, Oxford.

BENGTSSON, B. O. 1985. The flow of genes through a genetic barrier. In: Evolution Essays in honour of John Maynard Smith, Greenwood, J. J., Harvey, P. H. and Slatkin, M. (eds.) Cambridge University Press, Cambridge, pp. 31-42.

BENGTSSON, B. O. AND CHRISTIANSEN, F. B. 1983. A two-locus mutation-selection model and some of its evolutionary implications. Theoret. Pop. Biol, 24, 59-77.

CHARlesworth, B., LANDE, R. AND SLATKIN, M. 1982. A neo-Darwinian commentary on macroevolution. Evolution, 36, 474-498.

DOBZHANSKy, T. H. 1937. Genetics and the Origin of Species. Columbia University Press, New York.

FREDGA, K. 1986. Genetic differentiation in Sorex. IV. Localisation of a chromosomal hybrid zone in Sorex araneus, Hereditas, in press.

FRYKMAN, I. 1983. An electrophoretic study of genetic differentiation in Sorex. Ph.D. thesis, University of Lund, Sweden.

KARLIN, S. AND MCGREGOR, J. 1972. Application of the method of small parameters to multi-niche population genetic models. Theoret. Pop. Biol., 3, 210-238.

LANDE, R. 1981. Models of speciation by sexual selection on polygenic traits. Proc. Natl. Acad. Sci. (U.S.A.), 78, 37213725 .

LI, W. H. AND NEI, M. 1974. Stable linkage disequilibrium without epistasis in subdivided population. Theoret. Pop. Biol., 63, 173-183.

MAYNARD SMITH, J. 1983. The genetics of punctuation and stasis. Ann. Rev. Genet., 17, 11-26.

NAGYLAKI, T. 1975. Condition for existence of clines. Genetics, $80,595-615$.

NAGYLAKI, T. 1976. Clines with variable migration. Genetics, $83,867-886$.

Nei, M., Maruyama, T. AND wU, C. 1. 1983. Models of evolution of reproductive isolation. Genetics, 103, 557-579.

PETRY, D. 1983. The effect on neutral gene flow of selection at a linked locus. Theoret. Pop. Biol., 23, 300-313.

SLATKIN, M. 1975. Gene flow and selection in a two-locus system. Genetics, 81, 209-222.

SEARLE, J. B. 1984. Three new karyotypic races of the common shrew, Sorex araneus (Mammalia: Insectivara) and a phylogeny. Syst. Zool, 33, 184-194.

SPIRITO, F., ROSSI, C. AND RIZZONI, M. 1983. Reduction of gene flow due to the partial sterility of heterozygotes for a chromosome mutation. I. Studies on a "neutral" gene not linked to the chromosome mutation. Evolution, 37, 785-797.
SZYMURA, J. M. AND BARTON, N. H. 1986. Genetic analysis of a hybrid zone between the fire-bellied toads, Bombina bombina and $B$. variegata, near Cracow in Southern Poland. Evolution, in press.

WALSH, J. B. 1982. Rate of accumulation of reproductive isolation by chromosome rearrangements. Amer. Nat., 120, 510532.

White, M. J. D. 1968. Models of speciation. Science, 159, 10651070.

White, M. J. D. 1978. Modes of Speciation. W. H. Freeman, San Francisco.

WILLS, C. J. 1970. A mechanism for rapid allopatric speciation. Amer. Nat., 111, 603-605.

\section{APPENDIX: DERIVATIONS}

\section{Two demes: the rod model}

In this, the first and simplest model, we will consider two populations, geographically separated from each other, but exchanging a certain proportion of migrants in each generation. The role of a genetic barrier between the populations is studied by following the rate at which an allele at a marker locus increases in one of the populations. The migration rate is assumed to be small enough that the probability of crosses between hybrids can be ignored. All matings involving hybrids will therefore be with an individual of the native genotype We will begin by deriving a result on gene flow which is valid for arbitrary selection pressures. In the rest of the paper, the analysis will be restricted by the assumption that selection is weak in absolute magnitude; the results from this section will then be used primarily to check that the approximation of weak selection does not generally produce results that are misleading.

We consider a marker locus which lies at the end of a string of $n$ equally spaced loci (fig. 2(a)). We will call this configuration the "rod model". We assume that, at most, one crossover occurs in the region per meiosis. This assumption is not particularly restrictive. It is known that with positive interference between crossovers, it is rare for more than one crossover to occur over quite large regions of a chromosome. Genes which lie outside the region will be inherited more or less independently of the marker allele. The contribution to the barrier of all such independently inherited sets of loci can be calculated separately, and the total barrier is then the product of this barrier strength and that produced by the linked genes. (This follows from the result derived by Bengtsson (1985, pp. 35,36$)$.) The probability of a crossover occurring between any two adjacent loci is $r$, and the 
probability of a crossover between the marker locus and the nearest selected locus is $\alpha r$ (fig. 2(a)).

Since we assume that introgressing genes are rare, and that no more than one crossover occurs in each generation, the marker allele can only be found, in the recipient population, in linkage with a consecutive series of between 0 and $n$ introgressing, deleterious alleles, and paired with a chromosome from the native population. We label these genetic backgrounds $g_{0}, g_{1}, \ldots, g_{n}$. We also define $g_{n+1}$ as the population from which migrants come; this can be taken to be genetically homogeneous. (This numbering differs slightly from that used by Bengtsson (1985).) When a marker allele first enters the population, it will be in an F1 hybrid, and hence associated with background $g_{n}$. To calculate the rate at which it flows into the background of the native population, $g_{0}$, we define a matrix $L$, as above, whose elements $L_{i j}(i \neq j)$ give the numbers of genes which in the present generation are in background $g_{i}$, and will be in background $g_{j}$ in the next generation. Elements $L_{i i}$ give the number of genes which remain in background $i$, minus 1 . This matrix gives the rate of migration of genes between backgrounds, as well as between demes. The number of markers in a background carrying $i$ deleterious alleles that are transmitted to the next generation is $v_{i}$, the fitness of this background. $v_{0}$ is arbitrarily set to 1 . The probability that a marker will recombine away from all the deleterious alleles is $\alpha r$, and so $L_{10}=v_{i} \alpha r$ for $i=0$ to $n$. Similarly, elements $L_{i 1}$ to $L_{i(i-1)}=v_{i} r$, and $L_{i i}=\left(v_{i}(1-r(\alpha+i-1))-1\right)(i=0$ to $n)$. Since recombination with the native genotype can only reduce the number of introgressing genes associated with the marker, all further elements in row $i$ are zero. Finally, we must include physical movement of individuals between the demes, at a rate $m$. Since immigrants almost all mate with native homozygotes to give $F 1$-type hybrids, $L_{n+1, n}=m$. We are concerned here with movement in one direction only, and so can set $L_{n+1, n+1}=0$. All other elements in row $(n+1)$ are zero.

The flux of the marker allele into different genetic backgrounds is given by $\underline{\delta u}=\underline{u} \underline{L}$. The strength of the barrier to flow from $g_{n+1}$ into $g_{0}$ is defined as $b=m \Delta u / \delta u_{0}$. The vector of fluxes is zero, except for the extreme elements, which correspond to the influx from the donor deme $\left(\delta u_{n+1}\right)$, and the consequent efflux $\left(\delta u_{0}\right)\left(\delta u_{i}=0\right.$ for $i \neq 0$ or $n+1)$. We can suppose, without loss of generality, that the number of marker alleles in the recipient population $\left(u_{0}\right)$ is zero: increasing the allele frequency throughout by a constant would make no difference. Then, the matrix equation expands to:

$$
\begin{aligned}
\delta u_{0}= & \alpha r v_{1} u_{1}+\alpha r v_{2} u_{2} \cdots+\alpha r v_{n} u_{n} \\
0= & \delta u_{1} \\
= & {\left[(1-\alpha r) v_{1}-1\right] u_{1}+r v_{2} u_{2} \cdots+r v_{n} u_{n} } \\
0= & \delta u_{i} \\
= & {\left[(1-(\alpha+i-1) r) v_{i}-1\right] u_{i} } \\
& +r v_{i+1} u_{i+1} \cdots+r v_{n} u_{n} \\
0= & \delta u_{n-1} \\
= & {\left[(1-(\alpha+n-2) r) v_{n-1}-1\right] u_{n-1} \cdots+r v_{n} u_{n} } \\
0= & \delta u_{n}=\left[(1-(\alpha+n-1) r) v_{n}-1\right] u_{n} \cdots \\
& +m u_{n+1} \\
\delta u_{n+1}= & m\left(u_{n}-u_{n+1}\right) .
\end{aligned}
$$

Let $c_{i}=\sum_{j=i}^{n} u_{j} v_{j} ; u_{i} v_{i}=\left(c_{i}-c_{i+1}\right)$, and $u_{n} v_{n}=c_{n}$. Equation (A1) gives an explicit recursion for $c_{j}$ :

$$
\begin{aligned}
& 0=\delta u_{i} \\
&=\left[(1-(\alpha+i-1) r) v_{i}-1\right]\left(c_{i}-c_{i+1}\right) / v_{i}+r c_{i+1} \\
& \quad(\mathrm{~A} 2) \\
&\left.\left.\therefore c_{i}[1-(\alpha+i-1) r) v_{i}\right]=c_{i+1}[1-(\alpha+i) r) v_{i}\right] \\
& \quad(i \neq 0 \text { or } n) .
\end{aligned}
$$

According to the definition, the barrier strength is $b=m \dot{\Delta} u / \delta u_{0}=\delta u_{n} / \delta u_{0}$, where the latter expression conforms with the method used by Bengtsson (1985, equation (A2)) to calculate barrier strengths. From equation (5), $\delta u_{0}=\alpha r c_{1}$. Hence, the barrier strength generated by the set of $n$ selected loci is:

$$
\begin{aligned}
b & =\frac{m \Delta u}{\delta u_{0}}=\frac{\delta u_{n}}{\delta u_{0}} \\
& =\frac{\left(1-v_{n}(1-(\alpha+n-1) r)\right) c_{n}}{\alpha r c_{1} v_{n}} \\
& =\frac{\left(1-v_{n}(1-(\alpha+n-1) r)\right)}{\alpha r v_{n}} \\
& \times \prod_{i=1}^{n-1}\left(\frac{1-v_{i}(1-(\alpha+i-1) r)}{1-v_{i}(1-(\alpha+i) r)}\right) .
\end{aligned}
$$

In the special case where fitnesses are multiplicative $\left(v_{i}=(1-s)^{i}\right)$, this reduces to:

$$
b=\prod_{i=1}^{n}\left\{1+\frac{s}{\left(1-s-(1-s)^{i}(1-(\alpha+i-1) r)\right)}\right\} .
$$

When $S=n s$ and $R=n r$ are small, products and higher moments of these parameters can be 
ignored. The strength of the barrier can then be approximated by:

$$
b=\frac{\Gamma[n+(\alpha+\theta) /(1+\theta)] \Gamma[\alpha /(1+\theta)]}{\Gamma[n+\alpha /(1+\theta)] \Gamma[(\alpha+\theta) /(1+\theta)]} .
$$

Here, $\theta=S / R=s / r$ is a measure of the strength of selection against the chromosome region, relative to its overall recombination rate. This ratio will play an important part in the following analysis.

\section{Two demes: a marker locus embedded in a selected chromosome}

It is most unlikely that a marker gene would lie just at the end of a selected block of genes. It is more realistic, but also more difficult, to calculate the effective rate of gene flow for a locus embedded in a selected chromosome. To free itself from the selected alleles on either side, the marker allele we are considering must first recombine away from the set on one side, so that it is linked to the end of the remaining selected genes. A second recombination is then needed to free it completely; this second part of the process is identical to the case we considered above (fig. 2(b)).

In this more complicated case, we restrict attention to multiplicative fitnesses, and to weak selection and recombination (cf. equation (A5) above; $v_{i}=(1-s)^{i}$, ns and $\left.n r \ll 1\right)$. Let $u_{j, k}$ be the frequency of chromosomes carrying $j$ selected alleles to the left of the marker locus, and $k$ to the right (fig. 2(b)). Let the immigrant chromosome carry $J, K$ selected alleles to the left and right, respectively: $J+K=n$. The matrix $L$ is now a four dimensional structure which gives the probability that a marker allele which is in background $j, k$ in the present generation will be found in background $l, m$ in the next generation. Proceeding as before:

$$
\begin{aligned}
m \Delta u= & \delta u_{J, K}=-s(J+K) u_{J, K}-r(J+K-1) u_{J, K} \\
0= & \delta u_{j, k}=-s(j+k) u_{j, k}-r(j+k-1) u_{j, k} \\
& +r\left(\sum u_{1, k}+\sum u_{j, 1}\right) \\
0= & \delta u_{0, k}=-s k u_{0, k}-r(k-\alpha) u_{0, k} \\
& +r\left(\alpha \sum u_{1, k}+\sum u_{0,1}\right) \\
0= & \delta u_{j, 0}=-s j u_{j, 0}-r(j-1+\alpha) u_{j, 0} \\
& +r\left(\sum u_{1,0}+(1-\alpha) \sum u_{j, 1}\right) \\
\delta u_{0,0}= & r\left(\alpha \sum u_{1,0}+(1-\alpha) \sum u_{0,1}\right) .
\end{aligned}
$$

Let $c_{j, k}=\sum_{j}^{J} \sum_{k}^{K} u_{1, m}$. Then, for $j, k \geqq 1:$

$$
c_{j, k}=\frac{\left(\begin{array}{c}
\left(c_{j+1, k}+c_{j, k+1}\right)(j+k)(1+\theta) \\
-c_{j+1, k+1}((1+\theta)(j+k)+1)
\end{array}\right.}{((1+\theta)(j+k)-1)}
$$

Since $c_{J, K}=\Delta u$, the remaining $c_{j, k}$ can be found using this recurrence relation. Once the $c_{j, k}$ are known, $\delta u_{0,0}$ and hence $b$ can be calculated. We have found no closed expression for $b$ in this case; the barrier strength must be calculated numerically.

\section{Exchange across a continuous habitat}

In principle, extension of these results to a continuous habitat is straightforward. However, two problems arise. First, the recurrence relation for the $u_{j, k}$ (equation (A6)) must be replaced by a set of diffusion equations, which is harder to solve. Second, and more important, the gamete frequencies in the centre of a cline are intermediate, and so one must consider interactions between hybrid genotypes. This makes the system nonlinear, and a complete analytic solution becomes intractable. Moreover, the large number of possible haplotypes ( $2^{n}$ with $n$ loci) makes direct numerical solution impractical.

However, the problem can be solved for the limiting cases where selection on the chromosome region in question is much stronger, or much weaker, than recombination $(\theta \gg 1$ or $\theta \ll 1)$. This approach follows that of Barton (1983), where the accuracy of the approximations used is discussed in more detail. We assume that selection and recombination are weak $(S, R \ll 1)$, and that gene flow can be approximated by diffusion (equation (3)).

Selection stronger than recombination. When selection is much stronger than recombination introgressing chromosomes will be eliminated much faster than they can be broken up by recombination. The population of gametes will therefore consist almost entirely of the two parental types: $+++\cdot+++($ at frequency $p)$ or $---\cdot---$ (at frequency $q$ ). Here, "." represents the marker locus which is assumed, as above, to be embedded in the middle of the chromosome. For simplicity, we suppose that the marker is placed symmetrically ( $j=k=n / 2$, and $\alpha=\frac{1}{2}$; fig. 2(b)). This system can be approximately by selection $S$ against heterozygotes at a single locus, giving the following frequencies of the parental haplotypes (Bazykin, 
1969):

$$
\begin{aligned}
& p=(1+\tanh (2 x / w)) / 2+0(1 / \theta) \\
& q=(1-\tanh (2 x / w)) / 2+0(1 / \theta)
\end{aligned}
$$

(where $x$ is the position along the one-dimensional transect, and $w=\left(8 \sigma^{2} / S\right)^{1 / 2}$ is the cline width, defined as $1 /$ maximum gradient).

Consider now the flow of a marker allele $(\cdot)$ from the $+++\cdot+++$ background into the $---\cdot---$ background. This is most likely to occur in two stages. First, the original chromosome recombines in the region between the marker locus and one or other of the adjacent selected loci, to produce either $+++\cdot---$ or $---\cdot+++$ chromosome. Then, a second crossover must occur to complete the transfer (e.g., $\pm \pm \pm x \cdot===$ gives - - - - - ). More complicated sequences can be ignored, since they will require more crossovers, and so will give contributions of higher order in $(1 / \theta)$.

We must first find the frequency of singly recombinant chromosomes, disregarding for the moment the state of the marker locus. Call the combined frequency of $+++\cdot---$ and $---\cdot+++$ chromosomes $z$. Such chromosomes are produced by recombination at a rate $r$ from heterozygotes which are present at a frequency $2 p q$. They are almost always found in diploid combination with a parental chromosome, that is, in individuals with half their loci heterozygous. We assume that the two recombinant genotypes both have fitness $1-s(1+\gamma) / 4$ relative to the parental genotypes. This is consistent with the model of epistasis used above, in which the fitness of an individual is equal to $1-\operatorname{Sh}(\gamma+h(1-\gamma)$, where $h$ is the proportion of heterozygous loci. When $\gamma<1$, selection acts more strongly against heterozygotes when the proportion of heterozygous loci is high, whilst when $\gamma<1$, selection acts more strongly when the proportion of heterozygotes is low (fig. 5). However, we stress that no assumptions need to be made here about the fitnesses of any genotypes other than those described above. The mean fitness of the population is $1-2 S p q$, and so, at equilibrium, the distribution of singly recombinant chromosomes is given by:

$$
0=\frac{\sigma^{2}}{2} \frac{\partial^{2} z}{\partial x^{2}}-S[(\gamma+1) / 4-2 p q] z+2 r p q .
$$

We must now consider the frequency of the marker allele within each genetic background. Suppose, for convenience, that the two hybridising populations are almost fixed for alternate alleles $(-$ and +$)$ at some neutral marker locus. Because the population of singly recombinant chromosomes $(z)$ is generated by symmetrical recombination between the two parental chromosomes, the frequencies of the alternative markers within this background must be equal $\left(u_{z}=\frac{1}{2}\right)$. Now consider the flux of + marker alleles into the - - - - - (q) background from the pool of recombinants. Here, ". represents the marker locus, which can be in state + or - ). The frequency of heterozygotes between - - - $\cdot---$ and recombinant chromosomes is approximately $2 q z$; these recombine in the relevant region with probability $r / 2$. Half the products of such recombinations carry a marker allele derived from the recombinant background against a pure ---.-- background. The net flux into the latter background is therefore $2 q z \times(r / 2) \times(1 / 2) \times u_{z}=r q z / 4$. The $---\cdot---$ chromosomes make up a proportion $q$ of the population, and so the influx of marker alleles relative to the size of the receiving population in $(r q z / 4) / q=(r z / 4)$. We must now find the fate of this influx as it diffuses within the --..-background, and in particular, find the flux of marker alleles to the left, into the population well away from the barrier. The diffusion of neutral markers within the ---.--- genetic background can be described by a term $\left(\sigma^{2} / 2\right)\left(\partial^{2} u / \partial x^{2}\right)$. (The loss of markers by further recombinations with introgressing chromosomes is negligible.) However, we must also take account of the fact that the size of the population of $---\cdot---$ chromosomes $(q(x))$ varies from place to place. We can treat this density gradient in the same way as in Nagylaki (1976). The gradient in density $(\partial q / \partial x)$ causes a net flux of marker alleles, giving the second term in equation (A10a):

$$
\begin{aligned}
0 & =\frac{\sigma^{2}}{2} \frac{\partial^{2} u_{q}}{\partial x^{2}}+\frac{\sigma^{2}}{q} \frac{\partial q}{\partial x} \frac{\partial u_{q}}{\partial x}+\frac{r z}{4} \\
& =\frac{\sigma^{2}}{2 q^{2}} \frac{\partial}{\partial x}\left(q^{2} \frac{\partial u_{q}}{\partial x}\right)+\frac{r z}{4} .
\end{aligned}
$$

This equation can be integrated to give the gradient of $u=u_{q}$ outside the barrier, and hence the barrier strength in the limit of $\theta \gg 1$ is:

$$
\frac{1}{B_{-}}=\frac{(\partial u / \partial x)_{-}}{\Delta u}=\frac{1}{q^{2}} \int_{-\infty}^{\infty} \frac{r q^{2}}{2 \sigma^{2}} z d x .
$$

We have confirmed the above derivation by the more tedious procedure of writing explicit diffusion equations for each class of gametes in the population.

Selection weaker than recombination. When selection is much weaker than recombination $(\theta \ll 1)$, 
the approach used so far, based on following the frequency of a marker allele through different genetic backgrounds, becomes complicated. It becomes simpler to calculate the barrier strength by adding the effects of the pairwise linkage disequilibria between the marker locus and each locus involved in the selective maintenance of the genetic differences between the hybridising populations. These disequilibria will be of order $\theta$, whereas the higher order disequilibria which mediate interactions between multiple sets of selected loci, and the marker locus, will be of higher order in $\theta$, and may be neglected. The relation between a description in terms of gamete frequencies, and a description in terms of disequilibria is discussed by Barton (1983), where the expansion in powers of $\theta$ is given for a similar model to that dealt with here.

We outline here a general derivation of the barrier to gene flow in the limit of $\theta \ll 1$, which applies to any selection scheme in which fitnesses are not frequency dependent, and do not depend explicitly on geographic position. This derivation is given in detail in Barton (1986), where the relation between barriers produced by linkage disequilibrium, and by local reductions in density and dispersal, is discussed.

Changes in the overall frequency of the marker allele $(u)$ are caused by gene flow (equation (3)), and by selection at loci which are in linkage disequilibrium with the marker locus. Provided that the fitness of each genotype is independent of genotype frequency, the selection coefficient associated with each selected allele is proportional to the gradient in log mean fitness with respect to the allele. Hence:

$$
0=\frac{\sigma^{2}}{2} \frac{\partial^{2} u}{\partial x^{2}}+\sum_{i=1}^{n}\left(\frac{1}{2} \frac{\partial \log \bar{W}}{\partial p_{i}}\right) D_{i}+0\left(\theta^{2}\right) .
$$

Here, $p_{i}$ is the frequency of the " + " allele at the $i$ th selected locus. $D_{i}$ is the linkage disequilibrium between the $i$ th selected locus and the marker locus. For convenience, we assume that only two alleles segregate at each locus. We must now calculate these $D_{i}$. They are generated by the dispersal of parental combination of alleles into the centre of the cline $\left(\sigma^{2}\left(\partial p_{i} / \partial x\right)(\partial u / \partial x)\right.$; Li and Nei, 1974; Barton, 1979) and are broken down by recombination $\left(-r_{i} D_{i}\right.$, where $r_{i}$ is the rate of recombination between the marker and the $i$ th selected loci). Since the marker locus is neutral, no disequilibria are generated by selection. To leading order in $\theta, D_{i}=$ $\sigma^{2}\left(\partial p_{i} / \partial x\right)(\partial u / \partial x) / r_{i}$ (Barton, 1983, equation (B7)). If we assume that selection is spread evenly over the genome, so that the clines at loci a given map distance from the marker have similar gradients, then the individual recombination rates $r_{i}$ between the selected loci and the marker locus can be replaced by the hamonic mean recombination rate, $\bar{r}$. Equation (A12) becomes:

$$
\begin{aligned}
0= & \frac{\sigma^{2}}{2} \frac{\partial^{2} u}{\partial x^{2}} \\
& +\sum_{i}\left(\frac{1}{2} \frac{\partial \log \bar{W}}{\partial p_{i}}\right)\left(\frac{\sigma^{2}}{\bar{r}} \frac{\partial p_{i}}{\partial x} \frac{\partial u}{\partial x}\right)+\mathrm{O}\left(\theta^{2}\right) .
\end{aligned}
$$

This equation can be integrated to give an explicit expression for $u$ as a function of the mean fitness, $\bar{W}(x)$, since, when disequilibria are weak, $\log \bar{W}$ depends only on allele frequencies, and therefore $\left.\sum_{i=1}^{n}\left(\partial \log \bar{W} / \partial p_{i}\right)\left(\partial p_{i} / \partial x\right)=\partial \log \bar{W} / \partial x\right)$. Hence, equation (A13) becomes:

$$
\begin{aligned}
0 & =\frac{\sigma^{2}}{2} \frac{\partial^{2} u}{\partial x^{2}}+\frac{\sigma^{2}}{2 \bar{r}} \frac{\partial u}{\partial x} \frac{\partial \log \bar{W}}{\partial x} \\
& =\frac{\sigma^{2}}{2} \frac{\partial}{\partial x}\left(\bar{W}^{(1 / \bar{r})} \frac{\partial u}{\partial x}\right) .
\end{aligned}
$$

Integrating, equation (A14) gives:

$$
\frac{\partial u}{\partial x}=\frac{\partial u}{\partial x}-\left(\frac{\bar{W}(x)}{\bar{W}_{-}}\right)^{(-1 / \bar{r})}
$$

(Here, $\delta u_{-} / \delta x$ is the gradient to the left of the barrier, and $W_{-}$is the mean fitness to the left of the barrier.)

We can now integrate once more to find the change in marker frequency $(\Delta u)$ which is caused by selection on loci in linkage disequilibrium, and hence find the barrier strength (fig. 2(cj). As above, we assume that the barrier is symmetrical:

$$
\Delta u=\frac{\partial u_{-}}{\partial x} \int_{-\infty}^{\infty}\left[\left(\frac{-W(x)}{-W_{-}}\right)^{-(1 / \bar{r})}-1\right] d x
$$

and thus:

$$
\begin{aligned}
B & =\frac{\Delta u}{\left(\partial u_{-} / \partial x\right)} \\
& =\int_{-\infty}^{\infty}\left[\left(\frac{\bar{W}(x)}{\bar{W}}\right)^{(-1 / \bar{r})}-1\right] d x .
\end{aligned}
$$

An expression has thus been given (equation (A16a)) which relates the shape of clines for neutral or weakly selected alleles $(u(x))$ to the mean fitness $(\bar{W}(x))$. It could be used to make inferences about the mean fitness from the observed shape of clines at marker loci (e.g., Szymura and Barton, 1986). However, we are concerned here with the converse question: what is the effect of various types of selection on gene 
flow at other loci? To answer this question, we must find the spatial pattern of mean fitness, $\bar{W}(x)$, that is produced by various models of selection.

To simplify matters, we will assume that selection acts in a similar way at each locus, and furthermore, that the harmonic mean recombination rates between each locus and all the others are approximately the same, whichever locus is considered. The latter assumption will be accurate when a large number of loci is under selection $(\log (n) \gg 1)$, since the harmonic mean is then dominated by the influence of distant loci (see fig. 4(b), and below). Then, making the same assumptions as above:

$$
\begin{aligned}
0= & \frac{\sigma^{2}}{2} \frac{\partial^{2} p_{i}}{\partial x^{2}}+\sum_{j \neq i}\left(\frac{1}{2} \frac{\partial \log \bar{W}}{\partial p_{j}}\right)\left(\frac{\sigma^{2}}{r_{i j}} \frac{\partial p_{i}}{\partial x} \frac{\partial p_{j}}{\partial x}\right) \\
& +\frac{p_{i} q_{i}}{2} \frac{\partial \log \bar{W}}{\partial p_{i}}+\mathrm{O}\left(\theta^{2}\right) .
\end{aligned}
$$

Assuming that all $p_{i} \simeq p$ :

$$
\begin{aligned}
0= & \frac{\sigma^{2}}{2} \frac{\partial^{2} p}{\partial x^{2}}+\frac{\sigma^{2}}{2 \bar{r}}\left(\frac{\partial \log \bar{W}}{\partial x}\right)\left(\frac{\partial p}{\partial x}\right) \\
& +\frac{p q}{2} \frac{\partial \log \bar{W}}{\partial p}
\end{aligned}
$$

Multiplying through by $\partial p / \partial x$ :

$$
\begin{aligned}
0= & \frac{\sigma^{2}}{2} \frac{\partial}{\partial x}\left(\frac{1}{2}\left(\frac{\partial p}{\partial x}\right)^{2}\right) \\
& +\frac{\sigma^{2}}{2 r}\left(\frac{\partial p}{\partial x}\right)^{2}\left(\frac{\partial \log \bar{W}}{\partial x}\right)+\frac{p q}{2 n}\left(\frac{\partial \log \bar{W}}{\partial x}\right) .
\end{aligned}
$$

Since $\left(\partial \log \bar{W} / \partial p_{i}\right)\left(\partial p_{i} / \partial x\right)=(\partial \log \bar{W} / \partial x / n)$

$$
\begin{aligned}
\therefore \quad 0= & \frac{\sigma^{2}}{2} \frac{\partial}{\partial x}\left(\frac{\bar{W}^{(2 / \bar{r})}}{2}\left(\frac{\partial p}{\partial x}\right)^{2}\right) \\
& +\frac{p q}{2 n} \bar{W}^{(2 / r)} \frac{\partial \log \bar{W}}{\partial x} \\
\therefore \quad\left(\frac{\partial p}{\partial}\right)^{2}= & \frac{2 \bar{W}^{-(2 / \bar{r})}}{n \sigma^{2}} \int p q \bar{W}^{(2 / \bar{r})} d \log \bar{W} .
\end{aligned}
$$

In the case where selection acts multiplicatively against heterozygotes, so that $\bar{W}=\exp (-2 S p q)$, this equation can be integrated explicitly (Barton, 1983, equation 23):

$$
\left(\frac{\partial p}{\partial x}\right)^{2}=\frac{2 S}{n \sigma^{2}}\left(\frac{e^{4 \theta p q}-4 \theta p q-1}{2}\right) .
$$

(Where $\theta=S / r \simeq 2 S \log (n) / R$ when $\log (n) \gg 1$.)

In general, the barrier strength can be calculated by finding $(\partial p / \partial x)$ from equation (A20), and then substituting for $d x$ in equation (A16) to give an integral over $d p$. The resulting equation must usually be solved numerically, but for multiplicative selection against heterozygotes, an explicit solution can be found:

$$
\begin{aligned}
B & =\sqrt{\frac{\sigma^{2} n}{2 S}} \int_{0}^{1} \frac{\left(e^{2 \theta p q}-1\right) 2 \theta^{2} d p}{\sqrt{e^{4 \theta p q}-4 \theta p q-1}} \\
& =\sqrt{\frac{\sigma^{2} n}{2 S}} \sqrt{\frac{\theta}{2}} \int_{\sqrt{\theta}}^{\sqrt{\theta}} \frac{\left(e^{\theta / 2} e^{-t^{2}} / 2-1\right) d t}{\sqrt{ } e^{\theta} e^{-t^{2}}-\theta+t^{2}-1}
\end{aligned}
$$

(where $t=(2 p-1) \sqrt{\theta}$ ).

The integrand in the rightmost equation above equals 1 at $t=0\left(p=\frac{1}{2}\right), 1 / \sqrt{2}$ at $t= \pm \sqrt{\theta}(p=0$ or $1)$, and changes monotonically inbetween. The integral between $t=-\sqrt{\theta}$ and $+\sqrt{\theta}$ is therefore $2 F \sqrt{\theta}$, (where $1 / \sqrt{2}<F<1$ ), and so $(B / \sigma)=$ $F \theta \sqrt{n / S}=F \sqrt{n s} / r$. When $n \gg 1$, so that $r \simeq$ $R /(2 \log (n)),(B / \sigma) \simeq 2 F \log (n) \sqrt{n s} / R$. (If the number of loci is so large that $\theta=S / \bar{r} \gg 1, F$ equals 1. The formula given in Barton and Hewitt, 1983, p. 352 , applies in this regime, but is too large by a factor of $\sqrt{2}$.) 